\title{
TOPOVIBL-REC114 interaction regulates meiotic DNA double-strand breaks
}

Alexandre Nore ${ }^{1 *}$, Ariadna B Juarez-Martinez ${ }^{2 *}$, Julie Clément ${ }^{1}$, Christine Brun ${ }^{1}$, Boubou Diagouraga ${ }^{3}$, Corinne Grey ${ }^{1}$, Henri Marc Bourbon ${ }^{4}$, Jan Kadlec ${ }^{2 \$}$, Thomas Robert ${ }^{3 \$}$ and Bernard de Massy $^{1 \$}$

1: Institut de Génétique Humaine (IGH), Centre National de la Recherche Scientifique, Univ

Montpellier, Montpellier, France

2: Univ. Grenoble Alpes, CNRS, CEA, IBS, F-38000 Grenoble, France.

${ }^{3}$ : Centre de Biologie Structurale (CBS), CNRS, INSERM, Univ Montpellier, Montpellier, France

${ }^{4}$ : Centre de Biologie Intégrative, CNRS, Université de Toulouse, Toulouse, France

*: equal contribution

$\$$ : corresponding authors

Key words: meiosis, recombination, PAR, TopoVI, SPO11, TOPOVIBL, REC114, telomeres 


\section{Abstract}

Meiosis requires the formation of programmed DNA double strand breaks (DSBs), essential for fertility and for generating genetic diversity. In male and female meiotic cells, DSBs are induced by the catalytic activity of the TOPOVIL complex formed by SPO11 and TOPOVIBL. To ensure genomic integrity, DNA cleavage activity is tightly regulated, and several accessory factors (REC114, MEI4, IHO1, and MEI1) are needed for DSB formation in mice. How and when these proteins act is not understood. Here, we show that REC114 is a direct partner of TOPOVIBL, and identified their conserved interacting domains by structural analysis. We then analysed the role of this interaction by monitoring meiotic DSBs in female and male mice carrying point mutations in TOPOVIBL that decrease or disrupt its binding to REC114. In these mutants, DSB activity was strongly reduced genome-wide in oocytes, but only in subtelomeric regions in spermatocytes. Conversely, in mutant spermatocytes, DSB activity was delayed in autosomes. These results provide evidence that REC114 is a key member of the TOPOVIL catalytic complex, and that the REC114/TOPOVIBL interaction ensures the efficiency and timing of DSB activity by integrating specific chromosomal features. 


\section{Introduction}

The TOPOVIL complex is evolutionarily related to the TopoVI type IIB topoisomerase, and is composed of two conserved subunits: the SPO11 catalytic subunit and TOPOVIBL. TOPOVIBL shares homology with the GHKL-ATPase domain, with the central transducer domain and to a lesser extent, with the regulatory C-terminal domain (CTD) of the TopoVIB subunit of TopoVI ${ }^{1-6}$. The TOPOVIL meiotic-specific activity is finely regulated in time (i.e. must be turned on and off at precise time windows) and in space (i.e. active at specific chromosomal locations) during meiotic prophase. However, the molecular mechanisms underlying this complex regulation remain largely elusive. In mammals, DSB sites are determined by PRDM9 that recognizes specific DNA motifs and modifies chromatin upon binding to these sites ${ }^{7-9}$. Additional uncharacterized factors are then needed for SPO11 binding to or in proximity of PRDM9 binding sites and for inducing DSBs.

In the mouse, the accessory proteins REC114, MEI4, IHO1 and MEI1 are essential for DSB formation, and localize as foci on chromosome axes at meiotic prophase onset. It has been proposed that IHO1, MEI4 and REC114 directly control TOPOVIL through its recruitment or activation \{Dereli, 2021 \#12399;Kumar, 2010 \#5585;Kumar, 2015 \#9054;Kumar, 2018 \#10843;Stanzione, 2016 \#9769\}. MEI4 and REC114 form a stable complex, and a structural analysis revealed that REC114 N-terminus forms a Pleckstrin homology $(\mathrm{PH})$ domain ${ }^{10,11}$ harbouring exposed conserved residues potentially involved in protein-protein interactions. This suggests that REC114 acts as a regulatory platform. In line with this hypothesis, it was recently reported that ANKRD31 directly interacts with REC114 PH domain, and is involved in regulating DSB number and localization ${ }^{10,12}$. However, how these accessory proteins participate in TOPOVIL activity remains to be determined.

Here, using structural analysis, we found that the mouse REC114 PH domain directly interacts with a conserved C-terminal peptide of TOPOVIBL, identifying its CTD as a regulatory unit of the TOPOVIL complex. Accordingly, in mice where TOPOVIBL interaction with REC114 was disrupted, we observed meiotic DSB formation defects in both sexes. In females, DSB formation was drastically reduced. Conversely, in males, DSB formation was delayed, but DSB levels were not reduced excepted for sub-telomeric regions. On the $\mathrm{X}$ and $\mathrm{Y}$ chromosomes, which recombine specifically in the distal subtelomeric pseudo-autosomal region (PAR) region, DSB activity reduction led to chromosome synapsis defects. These results provide evidence that REC114 is part of the TOPOVIL catalytic complex. Moreover, this unprecedented sexual dimorphism suggests that REC114, through its interaction with TOPOVIBL, regulates DSB activity by sensing specific genomic features. 
bioRxiv preprint doi: https://doi.org/10.1101/2021.1130.470517; this version posted December 2, 2021. The copyright holder for this preprint (which was not certified by peer review) is the author/funder, who has granted bioRxiv a license to display the preprint in perpetuity. It is made available under aCC-BY-NC-ND 4.0 International license.

Results

\section{TOPOVIBL forms a stable complex with REC114}

Using yeast two-hybrid assays we identified a specific interaction between mouse TOPOVIBL and REC114. The deletion analysis showed that the N-terminal PH domain of REC114 was required for this interaction because TOPOVIBL did not bind to REC114 lacking the first 39 aa (40-259) (Fig. 1a, b, Supplementary Fig. 1). This interaction also required TOPOVIBL C-terminus as indicated by the absence of binding upon deletion of its last 29aa (TOPOVIBL 1-550) (Fig. 1a, c, Supplementary Fig. 1). We obtained similar results in vitro using Strep-tag pull-down assays (Fig. 1d, Extended Data Fig. 1a). While it was difficult to produce full-length TOPOVIBL, we could express and purify a 6xHisSUMO fusion TOPOVIBL protein that lacks part of its transducer domain (construct 1-385; Fig. 1d, lane 1) and also the 6xHis-TOPOVIBL CTD (450-579) (Fig. 1d, lane 2). REC114 clearly interacted with TOPOVIBL 450-579 (Fig. 1d, lane 5), but not with TOPOVIBL 1-385 (Fig. 1d, lane 4). REC114 N-terminal domain (REC114 1-159), which includes the PH domain, was sufficient for the interaction with TOPOVIBL 450-579 (Extended Data Fig. 1a, lane 2). In agreement, the REC114 PH domain and TOPOVIBL 450-579 co-eluted in a single peak during size exclusion chromatography (Extended Data Fig. 1b, Supplementary Fig. 2a).

The structure prediction analysis suggested that TOPOVIBL CTD did not to contain any known globular domain and was partially intrinsically disordered (Extended Data Fig. 1c). However, it indicated the presence of a putative helix at its C-terminus (Supplementary Fig. 3) that was better conserved among metazoan species compared with the rest of the sequence (Fig. 1e, Extended Data Fig. 1d). As our yeast two-hybrid assays suggested that the corresponding C-terminal 29 residues of TOPOVIBL (residues 551 to 579) were important for the interaction with REC114 (Fig. 1c), we hypothesized that this predicted conserved helix (residues 559-572) might represent the REC114 binding region. Using isothermal titration calorimetry (ITC), we showed that REC114 PH domain bound to TOPOVIBL CTD (450-579) with a dissociation constant (Kd) of $1.2 \mu \mathrm{M}$ (Extended Data Fig. 2a). Moreover, the interaction between a TOPOVIBL peptide spanning residues 559-576 and REC114 PH domain was in the same range (Kd: $3.3 \mu \mathrm{M})$ (Extended Data Fig. 2b). These results demonstrate that this highly conserved motif in TOPOVIBL CTD (559-576 aa) is sufficient for interaction with REC114.

We then determined the crystal structure of the complex formed by the REC114 PH domain (residues 15-159) and the TOPOVIBL 559-576 peptide by X-ray crystallography (2.2 resolution, $\mathrm{R}_{\text {free }}$ of $25.6 \%$, and R-factor of $23.1 \%$ ) (Supplementary Table S1). The structure of the TOPOVIBL-bound REC114 PH domain (two perpendicular antiparallel $\beta$-sheets followed by a C-terminal helix) was essentially the same as in its unbound form ${ }^{11}$. The TOPOVIBL C-terminal peptide folds into a single helix that interacts with the REC114 PH domain $\beta$-sheet formed of strands $\beta 1, \beta 2$ and $\beta 6$ - $\beta 8$, burying $731 \AA 2$ of surface area (Fig. 1f, Extended Data Fig. 2c). The interaction surface on REC114 is formed of highly conserved surface residues (Fig. 1g). In the N-terminal part of the TOPOVIBL peptide, L561, W562 and V566 pack against a hydrophobic surface of REC114 (Fig. 1h). Specifically, W562 is located 
in a hydrophobic pocked formed by aliphatic side chains of K95, V97, L104 and M115 of REC114. In the central part of the TOPOVIBL helix, L569 binds to a hydrophobic groove in REC114 formed by V97, R99, C102, L104, and R117 (Extended Data Fig. 2d). The well-conserved R99 and R117 residues form several hydrogen bonds with main-chain carbonyls in the TOPOVIBL helix, and R117 forms a salt bridge with E571. The C-terminal part of the TOPOVIBL peptide forms a $3_{10}$ helix where W572 inserts into another hydrophobic pocket of REC114 made of R24, V53, C102 and R117 (Extended Data Fig. 2e). W572 also forms cation- $\pi$ interactions by packing against the ammonium groups of the two arginine residues and also main-chain hydrogen bonds with R117 and Q119. Finally, L573 packs against a hydrophobic surface around M110 (Extended Data Fig. 2e).

Most of the TOPOVIBL residues involved in the interaction with REC114 are highly conserved across species (Fig. 1e, Extended Data Fig. 3a). We showed the interaction conservation by modelling the complex from different vertebrate species using AlphaFold ${ }^{13}$ (Extended Data Fig. 3b, c, d, e). To identify the TOPOVIBL residues required for the interaction with REC114, we mutated several candidate residues (W562, V566, L569 and W572) based on structure and conservation. In pull-down assays with the Strep-tagged REC114 PH domain, mutations in W562, V566 and L569 abolished the interaction (Fig. 1i, lanes 2-5), and the W572L mutation reduced the interaction compared with the wildtype residue (Fig. 1i, lane 6). We also mutated two hydrophobic residues of the REC114 $\beta$-sheet (V97D, L104D). V97D had little effect, whereas L104D abolished the interaction with TOPOVIBL (Fig. 1i, lanes 7,8). We obtained similar results in pull-down experiments using His-tagged TOPOVIBL (Extended Data Fig. 2f). We confirmed TOPOVIBL W562 role in the interaction by ITC measurement, because the peptide containing the W562A mutation did not bind to REC114 PH domain (Extended Data Fig. 2g). Gel filtration indicated that W562A in TOPOVIBL 450-579 did not disrupt the protein folding (Supplementary Fig. 4). Moreover, in yeast two-hybrid assays using full-length proteins we showed that W562A and W562G led to loss of any detectable interaction (Fig. 1j and Supplementary Fig. 5). We conclude that the TOPOVIBL W562A mutation specifically disrupts the interaction with REC114, and we selected this mutation for the in vivo studies (see below).

Mouse REC114 directly interacts also with MEI4 ${ }^{11}$ and ANKRD31 ${ }^{10}$. The REC114/MEI4 interaction does not involve the REC114 PH domain but the C-terminal domain (203-254) ${ }^{11}$. In agreement, we could show by size exclusion chromatography the simultaneous interaction of REC114 with MEI4 and TOPOVIBL (Extended Data Fig. 1d, Supplementary Fig. 2b). Conversely, the REC114/ANKRD31 interaction involves the REC114 PH domain and the key interacting residues are in similar positions and interact with the REC114 residues implicated in the binding with TOPOVIBL (Extended Data Fig. 2h, Supplementary Fig. 6a, b). By ITC, we observed that TOPOVIBL 450-479 did not bind to REC114 when REC114 was pre-saturated with ANKRD31 1808-1857 (Supplementary Fig. 6d). In pull-down assays, the interaction with TOPOVIBL 450-479 was reduced when REC114 was first bound to ANKRD31 1808-1857 (Extended Data Fig. 2i). These results indicate a mutually exclusive binding of TOPOVIBL and ANKRD31 to REC114 in vitro. 
bioRxiv preprint doi: https://doi.org/10.1101/2021.11.30.470517; this version posted December 2, 2021. The copyright holder for this preprint (which was not certified by peer review) is the author/funder, who has granted bioRxiv a license to display the preprint in perpetuity. It is made available under aCC-BY-NC-ND 4.0 International license.

\section{Top6bl mutations lead to a DSB activity reduction in oocytes}

To evaluate the biological significance of the TOPOVIBL-REC114 interaction, we generated two mutant alleles of mouse Top6bl: i) Top $6 b l^{W 562 A}$ harbouring the point mutation W562A that weakens the interaction between TOPOVIBL and REC114; and ii) Top $6 b l^{\Delta l 7 C t}$ harbouring a truncation of TOPOVIBL C-terminal helix that interacts with REC114 (Supplementary Fig. 7 for details on mutant generation).

We first investigated DSB formation during meiotic prophase of oocytes from embryonic ovaries (16 days post-coitum, dpc), when leptonema and zygonema are predominant ${ }^{14}$. To follow DSB formation, we analysed the phosphorylated form of $\mathrm{H} 2 \mathrm{AX}(\gamma \mathrm{H} 2 \mathrm{AX})$ that appears at chromatin domains around DSB sites upon DSB formation ${ }^{15}$. In wild-type oocytes, $\gamma \mathrm{H} 2 \mathrm{AX}$ was present over large chromatin domains at leptonema and zygonema and mostly disappeared at pachynema (Fig. 2a). In both Top6bl mutants, $\gamma \mathrm{H} 2 \mathrm{AX}$ intensity was strongly reduced at leptonema and zygonema: by 5.2- and 2.5fold, respectively, in Top $6 b l^{W 562 A / W 562 A}$, and by 8-fold and 7.6-fold, respectively, in Top $6 b l^{\Delta 17 C t / \Delta 17 C t}$ oocytes, compared with wild-type oocytes (Fig. 2a,b). To determine whether this could be due to delayed DSB formation, we monitored $\gamma \mathrm{H} 2 \mathrm{AX}$ levels at a later developmental stage (18dpc) in Top $6 b^{\Delta / 7 C t / \Delta 17 C t}$ mice and found a reduction by 7.8-fold (Supplementary Fig. 8a). These findings suggest that the two Top6bl mutations lead to a decrease of DSB activity.

We confirmed this hypothesis by quantifying DSB repair through the detection of DMC1 and RPA2. DMC1 binds to resected DSB ends and catalyses homologous strand exchange for DSB repair. RPA is recruited to resected DSB ends before and also after strand exchange during second end capture for repair ${ }^{16,17}$. In wild-type oocytes, we detected DMC1 and RPA foci that colocalized with the chromosome axis at leptonema and zygonema. The number of DMC1 foci was decreased in both Top6bl mutants at leptonema (3.1-fold in Top6bl $l^{W 562 A / W 562 A}$ and 18.5-fold in Top6bl ${ }^{417 C t / \Delta 17 C t}$ mice) (Fig. 2c, d).

DMC1 level in Top $6 b l^{\Delta 17 C t / \Delta 77 C t}$ mice was close to the background level because DMC1 foci at leptonema and zygonema were reduced by 22.9 -fold in Top $6 b^{-/}$mice where DSB formation is abolished (Fig. 2d). The reduction of DMC1 foci was comparable also at zygonema (Fig. 2d). We obtained similar results for RPA2 foci (Extended data Fig. 4a, b). In wild-type oocytes, DSB repair promotes interactions between homologues that are stabilized by the recruitment and assembly of several proteins, including SYCP1 and SYCP3, to form the synaptonemal complex (Fig. 2a) ${ }^{18}$. In both Top6bl ${ }^{W 562 A / W 562 A}$ and $T o p 6 b l^{\Delta 77 C t / 17 C t}$ mice, we observed only short stretches of synapsis and very few nuclei with full synapses. In 16dpc wild-type ovaries, $42.5 \%, 32.5 \%$ and $20 \%$ of oocytes were in leptonema, zygonema and pachynema $(\mathrm{n}=315)$, respectively, compared with $61.9 \%, 37.7 \%$ and $0.4 \%(\mathrm{n}=496)$ in Top $6 b l^{W 562 A / W 562 A}$, and $57.6 \%, 42.4 \%$ and $0 \%(\mathrm{n}=151)$ in $T_{0 p} 6 b l^{\Delta 17 C t / \Delta 17 C t}$ ovaries. Overall, these results are consistent with a reduced DSB activity that affects synapsis formation between homologues. 
As formation of meiotic DSBs depends on the pre-DSB proteins IHO1, REC114, MEI4, ANKRD31 and MEI1 10-12,19-21, and because the two Top6bl mutations disrupt the interaction with REC114, we assessed REC114 cytological localization in Top6bl $l^{W 562 A / W 562 A}$ and Top6bl $l^{\Delta 17 C t / \Delta 17 C t}$ mice. In wild-type oocytes, REC114, IHO1, MEI4 and ANKRD31 form several hundred foci on chromosome axes at leptonema and they progressively disappear as DSBs form ${ }^{10-12,21,22}$. The number of REC114 axis-associated foci was significantly higher in Top $6 b l^{W 562 A / W 562 A}$ and particularly in Top $6 b l^{\Delta 17 C t / \Delta 17 C t}$ mice at leptonema and especially at zygonema compared with wild-type oocytes (Fig. 2e, f). This is consistent with the decreased DSB activity, because REC114 foci are displaced from chromosome axes upon DSB formation ${ }^{11}$. We obtained similar results by analysing ANKRD31, MEI4 and IHO1 foci (Extended data Fig. 5, Supplementary Fig. 9). Overall, these analyses demonstrate that loading of REC114, ANKRD31, MEI4 and IHO1 foci is not altered by the Top $6 b l^{W 562 A}$ and Top $6 b l^{417 C t}$ mutations.

These meiotic prophase defects affected oogenesis: follicle number was strongly reduced in ovaries of Top $6 b l^{W 562 A / W 562 A}$ and particularly Top $6 b l^{\Delta 17 C t / \Delta 17 C t}$ mice. Indeed, Top $6 b l^{\Delta 17 C t / \Delta 17 C t}$ mice (Supplementary Fig. 10a, b, c) were sterile, whereas Top6bl ${ }^{\text {W562A/W562A }}$ mice were sub-fertile (Supplementary Fig. 10d). We conclude that the TOPOVIBL-REC114 interaction is essential for meiotic DSB formation in oocytes.

\section{In Top6bl spermatocytes, DSBs are delayed genome-wide and decreased in sub-telomeric regions}

Unlike female meiosis, DSB activity was efficient in spermatocytes from both Top $6 b l$ mutants, as indicated by the detection and quantification of $\gamma \mathrm{H} 2 \mathrm{AX}$ at late leptonema and zygonema (Fig. 3a, b, Extended data Fig. 6a,b). Conversely, at early/mid leptonema, $\gamma \mathrm{H} 2 \mathrm{AX}$ levels were lower in Top $6 b l^{W 562 A / W 562 A}$ and Top $6 b l^{\Delta 17 C t / \Delta 17 C t}$ than wild-type spermatocytes, suggesting a delay in DSB formation (Fig. 3b).

DMC1 and RPA2 foci also appeared later in both mutants compared with wild-type spermatocytes (Fig. 3c, Extended data Fig. 6a, c, d, e, f). The number of DMC1 and RPA2 foci was lower in Top $6 b l^{W 562 A / W 562 A}$ and Top $6 b l^{417 C t / \Delta 17 C t}$ than in wild-type spermatocytes, particularly at early/mid leptonema (8.7- and 3.7-fold reduction of DMC1 foci and 11.7- and 7.4-fold reduction of RPA2 foci in Top $6 b l^{W 562 A / W 562 A}$ and Top $6 b l^{\Delta 17 C t / \Delta 17 C t}$, respectively, compared with wild-type spermatocytes). Conversely, at zygonema and pachynema, the number of DMC1 and RPA2 foci was similar in wildtype and mutant spermatocytes (Fig. 3c, Extended data Fig. 6c, e, f). These findings suggest an efficient but delayed formation of DMC1 and RPA2 foci, and efficient DSB repair in Top6bl $l^{W 562 A / W 562 A}$ and

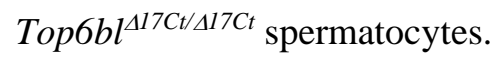

The localization of the DSB axis-associated proteins REC114, ANKRD31 and MEI4 at early/mid leptonema and the number of foci were similar or higher than in wild-type spermatocytes 
bioRxiv preprint doi: https://doi.org/10.1101/2021.11.30.470517; this version posted December 2, 2021. The copyright holder for this preprint (which was not certified by peer review) is the author/funder, who has granted bioRxiv a license to display the preprint in perpetuity. It is made available under aCC-BY-NC-ND 4.0 International license.

(Extended data Fig. 7, Supplementary Fig. 11). The number of foci gradually decreased from early/mid leptonema in wild type spermatocytes, but only after late leptonema (ANKRD31 and MEI4) or after early/mid leptonema but with a slower kinetic (REC114) in the Top6bl mutants (Extended data Fig. 7, Supplementary Fig. 11). These kinetic alterations are compatible with the observed delayed DSB activity because these axis-associated proteins disassemble from the axis upon DSB formation ${ }^{10-12}$.

To directly evaluate DSB activity and to map DSB sites, we monitored DMC1 enrichment by DMC1 chromatin- immunoprecipitation (ChIP), followed by ssDNA enrichment (DMC1- Single Strand DNA Sequencing, SSDS) ${ }^{23}$ in Top $6 b l^{\Delta 17 C t / \Delta 17 C t}$ mice. We identified 16780 DSB hotspots in Top $6 b l^{\Delta 17 C t / \Delta l 7 C t}$, among which 13261 (79\%) overlapped with wild-type hotspots (Fig. 3d). This indicated efficient PRDM9-dependent DSB localization in the mutant, as confirmed also by the absence of significant signal at PRDM9-independent hotspots ${ }^{24}$ (Fig. 3d, Extended data Fig. 8a, Supplementary Fig. 12a). The hotspot intensity was similar in Top $6 b^{417 C t / \Delta l 7 C t}$ and wild-type samples for most hotspots (Fig. 3e). However, a differential analysis of DMC1-SSDS signal intensity (using the DESeq2 R package, see Methods) showed that in Top $6 b l^{\Delta 17 C t / \Delta 17 C t}$ mice, the signal was decreased (by 1.2- to 24fold) in $12 \%$ of hotspots, and increased (by 1.45- to 3.3-fold) in $2 \%$ of hotspots (Extended data Fig. $8 \mathrm{~b}$ ). Visual analysis highlighted that in Top $6 b l^{\Delta 17 C t / \Delta 17 C t}$ mice, the DMC1-SSDS signal was decreased at hotspots located near the q-arm telomeres, the telomeres distal to centromeres (Fig. 3f and Supplementary Fig. 13, 14). We quantified this sub-telomeric phenotype by different approaches. First, by quantifying the ratio of the mutant/wild-type signal along the chromosome arms, we observed $a \geq 2$ fold decrease in the last few megabases proximal to the q-arm telomeres (Fig. 3g, Supplementary Fig. $12 \mathrm{~b})$. Second, in the ten hotspots closest to the q-arm telomeres, located within $4 \mathrm{Mb}$ of the telomere, DMC1-SSDS signal intensity at most hotspots for each chromosome was lower in Top $6 b l^{\Delta 17 C t / \Delta 17 C t}$ than wild-type samples (blue for autosomes, red for the X chromosome) (Fig. 3e). Third, quantification of this effect at each chromosome showed a reduced DMC1-SSDS signal within the ten q-arm telomeric adjacent hotspots in Top $6 b^{\Delta 17 C t / \Delta 17 C t}$ mice at most chromosomes (Fig. 3h, Supplementary Fig. 12c). Fourth, using DESeq, we evaluated the effect of the distance from telomeres, and found that the effect was most pronounced at hotspots located within $3 \mathrm{Mb}$ from telomeres and decreased when we tested larger intervals (Extended data Fig. 8c, d). In this analysis, the statistical significance also depended on the hotspot number in the tested region for each chromosome (see, for instance, chromosome 17, Supplementary Fig. 12). We conclude that in Top $6 b l^{\Delta 77 C t / \Delta 17 C t}$ mice, the DMC1-SSDS signal is specifically reduced in the $3 \mathrm{Mb}$ sub-telomeric region of the q-arm of most chromosomes. This finding could indicate a DSB decrease or higher DMC1 turnover. This region-specific alteration may not affect homologous interactions between autosomes because these interactions should be ensured by DSB sites along the chromosome arms. However, on the $\mathrm{X}$ and $\mathrm{Y}$ chromosomes, which depend on recombination in the PAR and which is located proximal to the telomere, the decreased DSB activity in this region in Top $6 b^{417 C t / \Delta 17 C t}$ mice (Fig.3f) could influence their homologous interaction. 
We tested this possibility by monitoring synapsis and bivalent formation on autosomes and on the $\mathrm{X}$ and $\mathrm{Y}$ chromosomes. Synapsis formation was normal on autosomes at pachynema in both mutants. However, the $\mathrm{X}$ and $\mathrm{Y}$ chromosomes were frequently unsynapsed in pachytene nuclei $(41 \%$ of Top $6 b l^{W 562 A / W 562 A}, 72 \%$ of Top $6 b^{417 C t / 477 C t}$ vs $18 \%$ in wild-type) (Fig. 3i, Extended data Fig. 9a), and also at metaphase (higher number of nuclei with $\mathrm{X}$ and $\mathrm{Y}$ univalents in Top6bl mutants) (Fig. 3j, k, Extended data Fig. 9b, c). We conclude that the decreased DMC1 signal on the X chromosome subtelomeric region is compatible with decreased DSB activity rather than with rapid DMC1 turnover. We propose that the TOPOVIBL-REC114 interaction is specifically required at sub-telomeric regions for full DSB activity and therefore, is essential for $\mathrm{X} / \mathrm{Y}$ chromosome synapsis and segregation.

These molecular and cytological phenotypes should have specific consequences on meiotic prophase and downstream events, because unsynapsed X/Y chromosomes induce metaphase I arrest ${ }^{25,26}$. Indeed, Top $6 b l^{W 562 A / W 562 A}$ and Top $6 b l^{4 l 7 C t / \Delta 17 C t}$ spermatocytes proceed through prophase like wildtype spermatocytes, but they arrested in metaphase and many cells were apoptotic, particularly in the $T o p 6 b l^{\Delta 17 C t}$ mutant. This metaphase I arrest was correlated with reduced sperm production in both mutants and fertility loss in Top $6 b l^{\Delta 17 C t / \Delta 17 C t}$ mice (Supplementary Fig. 15).

\section{Discussion}

A central question after the identification of the axis-associated proteins essential for meiotic DSB formation is to understand their function. Here, we found that TOPOVIBL CTD directly interacts with REC114 PH domain, and identified residues required for this interaction in vitro. We predict that TOPOVIBL can bind simultaneously to SPO11 through its transducer domain and to REC114 through the CTD. We used AlphaFold ${ }^{13}$ to model the SPO11/TOPOVIBL complex and included also REC114 (Extended data, Fig. 10). Compared with the TopoVI complex, the heterotetrameric model revealed the distinct property of TOPOVIBL compared with TopoVIB (lacking the N-terminal strap involved in dimerization, the conserved residues of the ATP binding site and the H2TH domain) and the conserved properties of SPO11 (dimerization-based catalytic site) (Extended data, Fig. 10). The REC114TOPOVIBL complex is connected by the flexible TOPOVIBL CTD to SPO11/TOPOVIBL core (Extended data, Fig. 10a). Although the exact REC114 effect on SPO11/TOPOVIBL organization/conformation is unknown, REC114 binding to TOPOVIBL could stabilize the SPO11/TOPOVIBL complex dimerization. This hypothesis is supported by the stoichiometry $(2: 1)$ of the Rec114/Mei4 complex in S. cerevisiae ${ }^{27}$ and by the observation that REC114 could bind simultaneously to TOPOVIBL and MEI4 (Extended data Fig. 1c). Nevertheless, REC114 interactions are certainly more complex because its PH domain also interacts with ANKRD31 ${ }^{10}$, thus potentially competing with TOPOVIBL (Extended Data Fig. 2, Supplementary Fig. 6, Supplementary discussion 1). Moreover, REC114 interacts with IHO1 in yeast-two hybrid assays ${ }^{21}$. The interplay of these different 
interactions remains to be determined, but disrupting the interaction between TOPOVIBL and REC114 may have additional consequences on other interactions.

The observation of meiotic DSB defects in female and male mice harbouring Top6bl mutations indicates that in vivo REC114 acts by directly interacting with the SPO11/TOPOVIBL complex. The stronger phenotype of mice carrying the Top $6 b l^{\Delta 17 C t}$ mutation (compared with Top $6 b l^{W 562 A}$ ) is explained by the absence of interaction in Top $6 b l^{417 C t}$, whereas the interaction is only weakened in Top $6 b l^{W 562 A}$. It also indicates that the C-terminal helix is the major interaction site between TOPOVIBL and REC114. Moreover, the distinct phenotypes show that REC114 is a regulatory subunit of the activity, and not just an accessory factor of the TOPOVIL complex. The reduced DSB activity in oocytes fits exactly the simple interpretation that REC114 binding to TOPOVIBL is required for the catalytic activity. The male phenotype highlights REC114 double role in fine tuning DSB activity by regulating the timing of DSB formation genome-wide and DSB formation at sub-telomeric regions. This implies that i) the TOPOVIBL-REC114 interaction is not essential for DSB activity in all genomic contexts. In A. thaliana, Rec114 role is dispensable ${ }^{28}$ and it would be interesting to test if the absence of Rec114 leads to a DSB delay in AtRec114 mutant; ii) REC114 activity senses or responds to specific chromosomal features. Sub-telomeric regions display specific features (i.e. nuclear organization during meiosis 29,30, architecture, organization and other epigenetic properties) that may influence the control of SPO11/TOPOVIBL activity. These regions behave differently from chromosome arms, based on their sensitivity to the expression of a GAL4BD-SPO11 fusion protein on DSB activity ${ }^{31}$, and to a lower DMC1/SPO11-oligonucleotides ratio ${ }^{32}$ (Supplementary discussion 4). Our data further emphasize the interest of analysing these chromosomal regions also because of their sex-specific meiotic recombination properties ${ }^{33,34}$.

Programming hundreds of DSBs in the genome is a challenge for the cell, and the current knowledge that the catalytic complex of SPO11/TOPOVIBL requires several other proteins is coherent with the need to regulate these events. Here, we described the central role of REC114 in SPO11/TOPOVIBL activity through its direct interaction with TOPOVIBL, thus highlighting a first level of this regulation. Moreover, the DSB program must be executed in two very different cell types (oocytes and spermatocytes), and our findings show that the REC114-TOPOVIBL interaction is sensitive to these differences. Other components of the meiotic DSB machinery and their potential multiple interactions should contribute to SPO11/TOPOVIBL regulation. Additional directedmutagenesis studies will unravel them and will identify the complex(es) active in vivo. 


\section{Mouse strains}

Mice were in the C57BL/6J background. Mice carrying the homozygous mutant alleles Top6bl<em1(W562A)BdM> and Top6bl<em2(delta17)BdM> were named Top6bl $l^{W 562 A / W 562 A}$ and Top $6 b l^{\Delta 17 C t / \Delta 17 C t}$, respectively. Top $6 b l^{-/}$mice carry the Gm960 ${ }^{\text {em2Arte }}$ allele, a null allele due to a $5 \mathrm{bp}$ deletion in Top $6 b l^{5}$. All experiments were carried out according to the CNRS guidelines and were approved by the ethics committee on live animals (project CE-LR-0812 and 1295).

\section{Generation of mutant mice by CRISPR/Cas9}

330 Mutant mice were created at the Jackson Laboratory using the CRISPR-Cas9 technology with three different guides and two different donor oligos (Table S4). Guides were selected to minimize off-target effects. The donor oligos were designed to change the W562 codon TGG (W) to GCG (A), and to introduce a silent mutation (A to G) to generate a Pst restriction site. The Top6bl<em1(W562A)BdM> allele, named $T o p 6 b l^{W 562 A}$, is the result of homologous recombination. The Top6bl<em2(delta17)BdM> allele, named Top $6 b l^{\Delta 17 C t}$, is the result of non-homologous repair and has a 4bp deletion (Supplementary Fig. 7). Founders were backcrossed with C57BL/6J animals to obtain heterozygous animals. The predicted TOPOVIBL protein expressed from each mutant allele and the genotyping strategies are shown in Supplementary Fig. 7.

\section{Yeast two-hybrid assays: clones, assays, western blotting}

All plasmids used in yeast two-hybrid assays were cloned with the Gateway® Gene Cloning Technology (Invitrogen) and transformed in the AH109 and Y187 haploid yeast strains (Clontech). AH109 and Y187 cells were transformed with Gal4 DNA binding domain (GBD) fusion plasmids derived from pAS2 and Gal4 activation domain (GAD) fusion plasmids that were obtained from pGAD. Purified colonies of diploid strains were streaked on synthetic medium (SD) plates lacking leucine and tryptophan (-LW), or leucine, tryptophan and histidine (-LWH), or leucine, tryptophan and histidine with $5 \mathrm{mM}$ aminotriazole (-LWH+3AT), or leucine, tryptophan, histidine and adenine (-LWHA). Dilution assays were performed by spotting cells on -LW, -LWH, -LWH+3AT and -LWHA plates that were incubated at $30^{\circ} \mathrm{C}$ for 3 days.

350 For verification of protein expression, protein extracts were prepared and analysed by western blotting, as previously described ${ }^{5}$, with anti-GAD (1:3000; UPSTATE-06-283) and anti-GBD (1:1000; SIGMA; G3042) antibodies.

\section{Preparation of mouse protein extracts, immunoprecipitation and western blotting}

Whole cell protein extracts were prepared from eight frozen testes collected at $14 \mathrm{dpp}$ for each genotype. After protein extraction by homogenizing cells with a Dounce homogenizer in HNTG buffer (150 mM 
$\mathrm{NaCl}, 20 \mathrm{mM}$ HEPES pH7.5, 1\% Triton X100, 10\% glycerol, $1 \mathrm{mM} \mathrm{MgCl}$, Complete protease Inhibitor (Roche 11873580001$)$ ), followed by sonication, benzonase $(250 \mathrm{U})$ was added at $4^{\circ} \mathrm{C}$ for $1 \mathrm{~h}$. After centrifugation $\left(16000 \mathrm{~g}, 4^{\circ} \mathrm{C}, 10 \mathrm{~min}\right)$ to remove debris, immunoprecipitation was performed with $5 \mu \mathrm{g}$ of homemade anti-TOPOVIBL antibody. For each immunoprecipitation, $3.5 \mathrm{mg}$ of whole cell protein extract and $50 \mu \mathrm{l}$ of Protein A Dynabeads (Invitrogen 10001D) were used. Then, immunoprecipitates were resuspended in $40 \mu 1$ of Laemmli buffer and TOPOVIBL immunoprecipitation was assessed by western blotting with a homemade affinity-purified anti-TOPOVIBL (1/1000) antibody followed by an anti-rabbit LC mouse monoclonal secondary antibody (Jackson ImmunoResearch 211-032-171).

\section{RT-PCR assays}

Total RNA was extracted with the miRNeasy Mini Kit (Qiagen) according to the manufacturer's instructions. For RT-PCR, first-strand DNA was synthesized using oligo d(T) $)_{18}$ (Ambion), SuperScriptIII (Invitrogen), and total RNA (1-2 $\mu \mathrm{g})$ from 16dpc ovaries.

The open reading frames of Top6bl and Spoll were amplified using standard PCR conditions and the primer pairs Oli63/Oli70 and Spo11:116U22/Spo11:655L22, respectively (Table 1). PCR cycling conditions were: $3 \mathrm{~min}$ at $94^{\circ} \mathrm{C}, 35 \mathrm{cycles}$ of $30 \mathrm{sec}$ at $94^{\circ} \mathrm{C}, 30 \mathrm{sec}$ at $54^{\circ} \mathrm{C}$, and $2 \mathrm{~min}$ or $30 \mathrm{sec}$ at $72^{\circ} \mathrm{C}$, followed by 5 min at $72^{\circ} \mathrm{C}$. Top $6 b l$ ORF was then digested with the EciI enzyme.

\section{Histological analysis of paraffin sections and TUNEL assay}

Mouse testes or ovaries were fixed in Bouin's solution for Periodic Acid Schiff (PAS) staining of testes and haematoxylin eosin staining of ovaries. Fixation was in $4 \%$ paraformaldehyde/1X PBS for immunostaining and TUNEL assay. Testes and ovaries were embedded in paraffin and cut in $3 \mu \mathrm{m}$-thick sections. Sections were scanned using the automated tissue slide-scanning tool of a Hamamatsu NanoZoomer Digital Pathology system. TUNEL assay was performed with the DeadEnd Fluorometric TUNEL System (Promega), according to the manufacturer's protocol.

\section{Spermatozoid counting}

After dissection of the epididymis caudal part from adult testes (2 month-old), spermatozoids were extracted from the epididymis by smashing or crushing the tissue in PBS. After homogenization by pipetting, $10 \mu 1$ of the soluble part was diluted in $1 \mathrm{~mL}$ of water, and spermatozoids were counted.

\section{Immunocytology}

Spread from spermatocytes and oocytes were prepared with the dry down technique, as described ${ }^{35}$, and immunostaining was performed as described ${ }^{36}$. 


\section{Antibodies}

Guinea pig anti-SYCP3 ${ }^{36}$, rabbit anti-SYCP1 (Abcam, 15090), rabbit anti-DMC1 (Santa Cruz, H100), anti-RPA2 (Abcam), anti-MEI4 ${ }^{19}$, anti-REC114 ${ }^{11}$, anti-REC114 (gift from S. Keeney), anti-ANKRD31 ${ }^{12}$, anti-IHO1 ${ }^{21}$, and mouse monoclonal anti-phospho-histone H2AX (Ser139) ( $\gamma \mathrm{H} 2 \mathrm{AX}$ ) (Millipore, 05636) antibodies were used for immunostaining. Homemade affinity purified anti-TOPOVIBL antibody: rabbits were injected with full-length mouse His-TOPOVIBL protein prepared from E. coli inclusion bodies. Rabbit serum was purified by affinity using His-TOPOVIBL purified from inclusion bodies.

\section{Metaphase spread preparation}

Tubules from decapsulated testes were pulled apart in $1 \%$ trisodium citrate and transferred into a $15 \mathrm{ml}$ tube. After pipette homogenization and 1min sedimentation, the cell-containing supernatant was transferred in a new $15 \mathrm{ml}$ tube. Following the same procedure, the tubule pieces were rinsed twice with $3 \mathrm{ml}$ of $1 \%$ trisodium citrate. The cell solution was centrifuged at $180 \mathrm{~g}$ for $10 \mathrm{~min}$, and the pellet resuspended in 100 $\mu \mathrm{l}$ of supernatant. Then, $3 \mathrm{ml}$ of methanol: acetic acid: chloroform (3:1:0.05) solution was added drop by drop to the cell solution (rolling the first drops down the sides of the tube while flicking the tube). Cells were then centrifuged at $180 \mathrm{~g}$ for $10 \mathrm{~min}$ and resuspended in $100 \mu \mathrm{l}$ of supernatant, and $3 \mathrm{ml}$ of methanol: acetic acid (3:1) was added to the tube. After 10min of incubation at room temperature, cells were centrifuged again (180g for $10 \mathrm{~min}$ ) and resuspend in $\sim 1 \mathrm{ml}$ methanol: acetic acid (3:1). To prepare the slides, $40 \mu 1$ of the cell suspension was dropped from a height of $\sim 40 \mathrm{~cm}$ onto a slide that was held titled at $45^{\circ}$. Slides were dried in a humid chamber.

\section{FISH for chromosome painting}

X (D-1420-050-FI; D-1420-050-OR) and Y (D-1421-050-FI; D-1421-050-OR) chromosome-specific probes were used according to the manufacturer (Metasystems Probes).

\section{Image analysis}

For focus quantification, all images were deconvoluted using the Huygens software. Image $\mathbf{J}$ was used to quantify foci that colocalized with the chromosome axis defined by SYCP3 staining.

For $\gamma \mathrm{H} 2 \mathrm{AX}$ quantification in oocytes, signal intensity was obtained using Cell Profiler on nondeconvoluted images. Integrated intensity was used for the analysis. For spermatocytes, both Cell Profiler and Image $\mathbf{J}$ quantifications were performed and gave similar results. The output of Image $\mathbf{J}$ integrated intensity is presented.

Staging criteria were as follows. Pre-leptotene nuclei had weak SYCP3 nuclear signal and no or very weak $\gamma \mathrm{H} 2 \mathrm{AX}$ signal; early leptotene nuclei were $\gamma \mathrm{H} 2 \mathrm{AX}$-positive and with only short SYCP3 fragments; mid leptotene nuclei were $\gamma \mathrm{H} 2 \mathrm{AX}$-positive and with short and long SYCP3 fragments; late leptotene were $\gamma \mathrm{H} 2 \mathrm{AX}$-positive and with only long SYCP3 fragments; zygotene nuclei had partially synapsed homologs; and pachytene cells had all 19 autosomes fully synapsed (Supplementary Fig. 16). 


\section{Statistical analysis}

The statistical analyses of cytological observations were done with GraphPad Prism 9. The nonparametric Mann-Whitney test was used to compare number of foci, and the Pearson's Chi square test to compare distributions, as indicated in the figure legends. The Chi square tests were performed at http://vassarstats.net/ and https://www.quantitativeskills.com/sisa/index.htm. Box plots (25-75 percentiles) show the median and 5-95 percentiles. Statistical tests for DMC1-SSDS data were done using R version 4.0.3. All tests and p-values (n.s., not significant. $* \mathrm{P}<0.05$, **P $<0.01$, *** $\mathrm{P}<0.001$, $* * * * \mathrm{P}<0.0001)$ are provided in the corresponding legends and/or figures.

\section{DMC1-SSDS analysis}

\section{Library preparation and sequencing}

DMC1 ChIP-seq was performed as described in ${ }^{37}$ using a goat anti-DMC1 antibody (Santa Cruz, C20). Six Top $6 b l^{\Delta 77 C t / \Delta 17 C t}$ testes and two wild-type testes from 12 to 25 -week-old mice were used for each replicate. Sequencing was performed on a HiSeq 2500 instrument in paired-end mode (2x150bp).

DMC1-SSDS mapping and hotspot identification

After quality control and read trimming to remove adapter sequences and low-quality reads, DMC1 ChIP-SSDS reads were mapped to the UCSC mouse genome assembly build GRCm38/mm10. The previously published method ${ }^{23}$ was used for DMC1-SSDS read mapping (i.e. the BWA modified algorithm and a customized script that were specifically developed to align and recover ssDNA fragments). A filtering step was performed on the aligned reads to keep only non-duplicated and highquality uniquely mapped reads with no more than one mismatch per read. To identify meiotic hotspots from biologically replicated samples in DMC1-SSDS, the Irreproducible Discovery Rate (IDR) method was used, as done in our previous studies. This method was developed for ChIP-seq analysis and extensively used by the ENCODE and modENCODE projects ${ }^{38}$. The framework developed by Qunhua $\mathrm{Li}$ and Peter Bickel's group (https://sites.google.com/site/anshulkundaje/projects/idr) was followed. Briefly, this method allows testing the reproducibility within and between replicates by using IDR statistics. Following their pipeline, peak calling was performed using MACS version 2.0.10 with relaxed conditions (--pvalue=0.1 --bw1000 --nomodel --shift400) for each of the two replicates, the pooled dataset, and pseudo-replicates that were artificially generated by randomly sampling half of the reads twice for each replicate and the pooled dataset. Then IDR analyses were performed and reproducibility was checked. Final peak sets were built by selecting the top $\mathrm{N}$ peaks from pooled datasets (ranked by increasing $\mathrm{p}$ values), with $\mathrm{N}$ defined as the highest value between $\mathrm{N} 1$ (the number of overlapping peaks with an IDR below 0.01, when comparing pseudo-replicates from pooled datasets) and N2 (the number of overlapping peaks with an IDR below 0.05 when comparing the true replicates, as recommended for the mouse genome). Hotspot centring and strength calculation were computed following the method described by Khil et al. ${ }^{23}$. All read distributions and signal intensities presented in this work were calculated after pooling reads from both replicates, if not otherwise stated. When DSB maps were 
bioRxiv preprint doi: https://doi.org/10.1101/2021.11.30.470517; this version posted December 2, 2021. The copyright holder for this preprint (which was not certified by peer review) is the author/funder, who has granted bioRxiv a license to display the preprint in perpetuity. It is made available under aCC-BY-NC-ND 4.0 International license.

compared between mouse genotypes, the $1 \mathrm{bp}$-overlaps were restricted to the central $400 \mathrm{bp}$ of hotspots (+/- 200bp around the peak centre). For correlation plots, the type 1 single-strand DNA signal was library-normalized (fragment per million).

\section{Differential analysis of hotspot strength}

To compare hotspot usage between Top $6 b^{417 C t / \Delta 17 C t}$ and wild-type mice, DMC1-SSDS signal intensity was compared at the 13562 wild-type hotspots using DESeq ${ }^{39}$. Among these hotspots, 4207 hotspots (31\%) were filtered out with the default independent filtering option using the mean of normalized counts as filter statistic. The aim was to remove sites with too low counts (mean count below 22) that have zero or low chance of showing significant differences to increase the detection power for the other sites. For the 9355 tested hotspots, log fold change shrinkage was performed to correct data dispersion using the apeglm method ${ }^{40}$. The hotspots with increased or decreased DMC1-SSDS signal intensity were then determined using an adjusted p-value threshold of 0.1 and a $\log 2$ fold change value below or above zero, respectively. This led to the identification of 214 increased (1.2\% of total hotspots, and $2 \%$ of the tested ones), 1098 decreased (8\% of total hotspots, and $12 \%$ of the tested ones), and 8043 unchanged hotspots in Top $6 b l^{\Delta 17 C t / \Delta 17 C t}$ mice (Extended Data Fig. 8b).

\section{Analysis of hotspot distribution at sub-telomeric regions}

Visual inspection of DMC1-SSDS signal intensity along chromosomes and the localization of hotspots with decreased DMC1-SSDS signal intensity in Top $6 b l^{\Delta 17 C t / \Delta 77 C t}$ suggested that much of the DMC1 signal decrease was located near the q-arm telomeres (telomeres distant from centromeres). To test whether this biased distribution was significant, each hotspot was annotated as sub-telomeric when within the sub-telomeric region defined with a variable size from 1 to $10 \mathrm{Mb}$. For each sub-telomeric region, the numbers of unchanged, decreased and increased hotspots in the sub-telomeric versus the non-sub-telomeric region (i.e. the rest of the chromosome) were counted. Pearson's Chi-square tests were computed (by taking into account or not the increased hotspots) and p-values were adjusted for multiple testing using the Benjamini \& Yekutieli method. Megabase-normalized counts (hotspot density) were measured and plotted (Extended Data Fig. 8c for sub-telomeric regions of 3Mb). For each chromosome, the sub-telomeric over non-sub-telomeric ratio of hotspot density was calculated for each unchanged, decreased or increased hotspot category (Extended Data Fig. 8d). Alternatively, to evaluate hotspot activity without a fixed distance from the telomere, for each chromosome, the

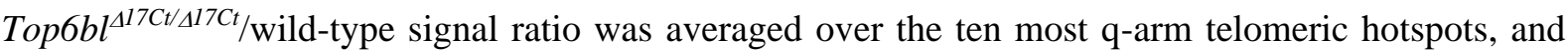
then compared to the averaged signal ratio measured over another set of ten consecutive hotspots randomly chosen along the chromosome (excluding the last tens). Then, the ratio of these two mean values was computed. The procedure was repeated 10 times, each time with a different random set of 10 non-sub-telomeric hotspots. The sub-telomeric effects are presented in Fig. 3h as the distribution of these ratios. As control, these ratios were computed not between the last ten and ten non-sub-telomeric 
hotspots, but between two non-sub-telomeric random hotspot sets (procedure repeated 10 times) (Supplementary Fig. 12c).

The differential and sub-telomeric analyses were performed to compare Ankrd31 $1^{-/}$and $A n k r d 31^{+/+}$ DMC1-SSDS signals at wild-type hotspots using our previously published data ${ }^{12}$. Only DMC1-SSDS signal variations at PRDM9-dependent hotspots were considered for this differential analysis and not the subset of default hotspots (activated in $\operatorname{Prdm} 9 \mathrm{KO}$ mice) that is activated in $\mathrm{Ankrd} 3 \mathrm{I}^{-/}$mice.

\section{Data deposition}

The DMC1-SSDS raw and processed data for this study have been deposited under a confidential status in the European Nucleotide Archive (ENA) at EMBL-EBI (accession number PRJEB43730) (https://www.ebi.ac.uk/ena/browser/view/PRJEB43730) and will be released as soon as the present paper is accepted for publication. Data are available upon request during the reviewing process.

\section{Protein expression, purification and crystallization}

His-tagged mouse REC114 15-159 was expressed in E. coli BL21-Gold (DE3) cells (Agilent) from the pProEXHTb expression vector (Invitrogen). The protein was first purified by affinity chromatography using the $\mathrm{Ni}^{2+}$ resin. After His-tag cleavage with the TEV protease, it was purified through a second $\mathrm{Ni}^{2+}$ column and size-exclusion chromatography. The pure protein was concentrated to $20 \mathrm{mg} \mathrm{ml}^{-1}$ in a buffer containing $20 \mathrm{mM}$ Tris, $\mathrm{pH}$ 8.0, $200 \mathrm{mM} \mathrm{NaCl}$ and $5 \mathrm{mM} \beta$-mercaptoethanol, and supplemented with a three-fold molar excess of TOPOVIBL peptide (559-EDLWLQEVSNLSEWLNPG-576). The complex was crystallized using the hanging drop vapor diffusion method at $20^{\circ} \mathrm{C}$. The best diffracting crystals grew within seven days in a solution containing 1.6M MgSO $4,100 \mathrm{mM}$ MES (pH 6.5), and 10\% (v/v) dioxane. For data collection at $100 \mathrm{~K}$, crystals were snap-frozen in liquid nitrogen with a solution containing mother liquor and $25 \%$ (vol/vol) glycerol.

\section{Data collection and structure determination.}

Crystals of the mouse REC114-TOPOVIBL complex belong to the space group P6 22 with the unit cell dimensions $a, b=108.6 \AA$ and $c=83.4 \AA$. The asymmetric unit contains one REC114-TOPOVIBL dimer and has a solvent content of $68 \%$. A complete dataset was collected to a resolution of $2.2 \AA$ on the ESRF beamline ID30B. Data were processed using XDS ${ }^{41}$. Phases were obtained by molecular replacement using PHASER ${ }^{42}$ with the crystal structure of the REC114 PH domain (PDB code: 6HFG) as search model. The initial map was improved using the prime-and-switch density modification option of RESOLVE ${ }^{43}$. After manual model rebuilding with $\mathrm{COOT}^{44}$, the structure was refined using Refmac5 ${ }^{45}$ to a final $R$-factor of $23.2 \%$ and $R_{\text {free }}$ of $25.6 \%$ (Table $\mathrm{S} 1$ ) with all residues in the allowed $(97.5 \%$ in favored) regions of the Ramachandran plot, as analysed by MOLPROBITY ${ }^{46}$. A representative part of the $2 F_{\mathrm{o}}-F_{\mathrm{c}}$ electron density map covering the TOPOVIBL-REC114 interface is shown in Supplementary Fig. 6e. 


\section{Pull-down assays}

Full-length REC114 and its variants were cloned as Strep-tag fusions into pRSFDeut-1 (Novagen). TOPOVIBL 1-385 was cloned as a 6xHis-SUMO fusion protein in pETM11, and TOPOVIBL 450-579 and its mutated versions as 6xHis fusion proteins in pProEXHTb. Proteins were expressed individually in E. coli BL21Gold (DE3) cells. For analysis of the structure-based mutants, half of each culture was used for Strep-tag pull-down assays and the other half for His-tag pull-down assays. For Strep-tag pulldown assays, TOPOVIBL proteins were affinity-purified using $\mathrm{Ni}^{2+}$ resin. Following cell disruption, the Strep-REC114-containing supernatant was added to a Strep-Tactin XT resin that was then extensively washed. The REC114-bound resin was then divided for individual pull-down experiments. Equal amounts of TOPOVIBL proteins were added and incubated with the REC114-bound resin with agitation at $5^{\circ} \mathrm{C}$ for $10 \mathrm{~min}$. Columns were then washed with a buffer containing $100 \mathrm{mM}$ Tris $\mathrm{pH} 8,150 \mathrm{mM}$ $\mathrm{NaCl}, 5 \mathrm{mM} \beta$-mercaptoethanol. Bound proteins were eluted by addition of $50 \mathrm{mM}$ of D-Biotin, and analysed on $15 \%$ SDS-PAGE. The His-tag pull-down experiments were performed in a similar way, but REC114 proteins were first purified on Strep-Tactin XT resin and then added in equal amounts to the TOPOVIBL-bound $\mathrm{Ni}^{2+}$ resin.

\section{Competition assay}

TOPOVIBL 450-579 was purified using $\mathrm{Ni}^{2+}$ resin as described above. ANKRD31 1808-1857 was cloned as 6xHis-MBP fusion in pETM41. Strep-REC114 1-159 and ANKRD31 1808-1857 were individually expressed in E. coli BL21Gold (DE3) cells. Following cell disruption, supernatants containing soluble Strep-REC114 1-159 and ANKRD31 1808-1857 were mixed and loaded onto StrepTactin XT resin. The resin bound with the REC114 1-159-ANKRD31 1808-1857 complex was then extensively washed and divided into $1 \mathrm{ml}$ fractions for individual competition assays by adding an increasing amount of purified His-tagged TOPOVIBL 450-579 (1mg, 5mg, 10mg and 20mg). After 10 min incubation at $5^{\circ} \mathrm{C}$, columns were washed with a buffer containing $100 \mathrm{mM}$ Tris $\mathrm{pH} 8,150 \mathrm{mM} \mathrm{NaCl}$, $5 \mathrm{mM} \beta$-mercaptoethanol, and bound proteins were eluted by adding $50 \mathrm{mM}$ of D-Biotin, and analysed on $12 \%$ Tris-Tricine SDS PAGE.

\section{Isothermal Titration Calorimetry (ITC)}

ITC experiments were performed at $25^{\circ} \mathrm{C}$ using an ITC200 microcalorimeter (MicroCal). Experiments included one $0.5 \mu \mathrm{l}$ injection and $18-20$ injections of $1.5-2 \mu \mathrm{L}$ of $0.3-1.8 \mathrm{mM}$ TOPOVIBL (TOPOVIBL 450-579, TOPOVIBL 559-576 or TOPOVIBL 559-576 W562A) into the sample cell that contained 30$40 \mu \mathrm{M}$ REC114 15-159 in $20 \mathrm{mM}$ Tris ( $\mathrm{pH} 8.0$ ), $200 \mathrm{mM} \mathrm{NaCl}, 5 \%$ glycerol and $5 \mathrm{mM} \beta$ mercaptoethanol. The initial data point was deleted from the data sets. Binding isotherms were fitted with a one-site binding model by nonlinear regression using the Origin software, version 7.0 (MicroCal). 
bioRxiv preprint doi: https://doi org/10.1101/2021.1130.470517: this version posted December 2 2021. The copyright holder for this preprint (which was not certified by peer review) is the author/funder, who has granted bioRxiv a license to display the preprint in perpetuity. It is made available under aCC-BY-NC-ND 4.0 International license.

\section{Acknowledgments:}

We thank the following Biocampus facilities from Montpellier for their service: the Réseau des Animaleries de Montpellier (RAM) for animal care, the Réseau d'Histologie Expérimentale de Montpellier (RHEM) for histology, and Montpellier Resources Imagerie (MRI) for microscopy. We thank all lab members for discussion and Pauline Auffret for support in bioinformatic analysis. We thank Scott Keeney for the anti-REC114 antibody, Attila Toth for the anti-ANKRD31 and anti-IHO1 antibodies. We thank Caroline Mas for assistance with ITC and the staff of the ESRF-EMBL (European Synchrotron Radiation Facility-European Molecular Biology Laboratory) Joint Structural Biology Group, particularly Andrew McCarthy, for access to and help with the ESRF beamlines. We thank the EMBL high-throughput crystallization facility (HTX).

Funding: BdM was funded by ANR Topobreaks (ANR- 18-CE11-0024-01), Prize Coups d'Élan for French Research from the Fondation Bettencourt-Schueller, ERC (European Research Council (ERC)

Executive Agency under the European Community's Seventh Framework Programme (FP7/2007-2013 Grant Agreement no. [322788])), CNRS and the CNRS ATIP-Avenir program funding to JK.

Ariadna B. Juarez-Martinez was supported by the Labex GRAL (Grenoble Alliance for Integrated Structural Cell Biology) (ANR-10-LABX-49-01) and the People Programme (Marie Curie Actions) of the European Union's Seventh Framework Programme (FP7/2007-2013) under REA grant agreement n. PCOFUND-GA-2013-609102, through the PRESTIGE programme coordinated by Campus France. This work used the platforms of the Grenoble Instruct-ERIC center (ISBG; UAR 3518 CNRS-CEAUGA-EMBL) within the Grenoble Partnership for Structural Biology (PSB), supported by FRISBI (ANR-10-INBS-0005-02) and GRAL, financed within the University Grenoble Alpes graduate school (Ecoles Universitaires de Recherche) CBH-EUR-GS (ANR-17-EURE-0003). 


\section{Figure legends}

\section{Fig. 1. TOPOVIBL-REC114 interaction and complex.}

a. Schematic representation of the domain structure of mouse REC114 (left) and TOPOVIBL (right). PH: Pleckstrin homology domain, MBR: MEI4 Binding Region, GHKL: Gyrase, HSP90, Histidine Kinase, MutL domain, L: linker, C-term: C-terminal.

b. Yeast two-hybrid assays showed that REC114 N-terminal domain is required for the interaction with TOPOVIBL. Growth (+ or -) was assayed on medium lacking leucine, tryptophane and histidine and with 5mM 3-amino-1,2,4-triazole. Additional assays are in Supplementary Fig. 1.

c. Yeast two-hybrid assays indicated that TOPOVIBL C-terminal region is required for the interaction with REC114. Growth (+ or -) was assayed on medium lacking leucine, tryptophane and histidine. Additional assays are in Supplementary Fig. 1.

d. Strep-tag pull-down experiments with REC114 and TOPOVIBL. The indicated TOPOVIBL domains were first purified on $\mathrm{Ni}^{2+}$ columns (lanes 1,2) and then loaded onto Strep-Tactin columns with or without previously bound full-length Strep-REC114. TOPOVIBL 1-385 is not retained by REC114 (lane 4), whereas TOPOVIBL C-terminal region (450-579) is sufficient for the interaction with REC114 (blue star, lane 5).

e. Sequence alignment of the 14-aa conserved motif at TOPOVIBL C-terminus in metazoans (see Extended Data Fig. 1 for the full alignment). Brown letters: equivalent amino acids; white letters: identical amino acids.

f. Ribbon representation of the overall structure of the REC114-TOPOVIBL complex. The REC114 PH domain is in blue, and the TOPOVIBL peptide in brown. Alpha helices $(\alpha)$ and beta sheets $(\beta)$ are labelled.

g. Surface representation of REC114 to highlight the conserved surface residues. Sequence conservation is represented from grey to blue according to the colour scale bar below. TOPOVIBL is shown as a cartoon and the key interacting residues as sticks.

h. Details of the interaction between the N-terminal part of the TOPOVIBL helix (brown) and REC114 (blue). TOPOVIBL W562 inserts into a hydrophobic pocket on the $\beta$-sheet formed by strands $\beta 1$ and $\beta 6-\beta 8$.

i. SDS-PAGE analysis of Strep-tag pull-down experiments with the indicated TOPOVIBL and REC114 proteins. Wild-type (WT) and mutants of His-TOPOVIBL were first purified on $\mathrm{Ni}^{2+}$ column and loaded on Strep-REC114 bound Strep-Tactin resin.

j. Essential role of TOPOVIBL W562 in the interaction with REC114 shown by yeast two-hybrid assays. Growth (+ or -) was assayed on medium lacking leucine, tryptophane and histidine and with 5mM 3amino-1,2,4-triazole. 
Fig. 2. In Top6bl $l^{W 562 A / W 562 A}$ and Top6bl ${ }^{\Delta 17 C t / \Delta 17 C t}$ mice, meiotic DSB activity is decreased in oocytes.

a. Immunostaining of $\gamma \mathrm{H} 2 \mathrm{AX}, \mathrm{SYCP} 3$ and SYCP1 in oocytes from $16 \mathrm{dpc}$ wild-type (+/+), Top $6 b l^{W 562 A / W 562 A}$, and Top $6 b l^{417 C t / \Delta 17 C t}$ ovaries. Scale bar, $10 \mu \mathrm{m}$.

b. Quantification of $\gamma \mathrm{H} 2 \mathrm{AX}$ signal intensity in leptotene and zygotene (or zygotene-like) nuclei of oocytes from $16 \mathrm{dpc}$ wild-type (+/+ or Top $\left.6 b l^{+/ \Delta 77 C t}\right)$, Top $6 b l^{W 562 A / W 562 A}, T_{o p} 6 b l^{477 C t / \Delta 77 C t}$, and Tob6blmice ( $\mathrm{n}=5,2$, 2, and 1 mouse/genotype). Number of nuclei at leptonema: 126, 123, 73, and 23; number of nuclei at zygonema: 241, 132, 64, and 33 for each genotype. Ratios (mean \pm SD) of the integrated intensity between the mean values in mutant nuclei and in wild-type nuclei are plotted. P values were determined using the two-tailed unpaired Mann-Whitney test. The fold reduction is the ratio of the wildtype to mutant mean values.

c. Immunostaining of DMC1 and SYCP3 in oocytes from $16 \mathrm{dpc}$ or $17 \mathrm{dpc}$ wild-type (+/+), Top6bl $l^{W 562 A / W 562 A}$ and Top6bl ${ }^{417 C t / \Delta 17 C t}$ ovaries. Scale bar, $10 \mu \mathrm{m}$.

d. Quantification of DMC1 foci. DMC1 axis-associated foci were counted in leptotene and zygotene

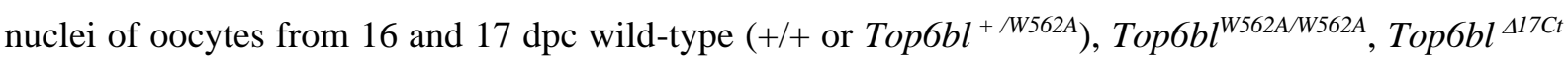
${ }^{1} \triangle 17 C t$, and $T o p 6 b l^{-}$mice. Number of nuclei at leptonema: 50, 8, 22, and 28; number of nuclei at zygonema: 79, 28, 29, and 27 for each genotype, respectively. Grey bars show the mean values. P values were determined using the two-tailed unpaired Mann-Whitney test. The fold difference relative to wildtype is shown.

e. Immunostaining of REC114 and SYCP3 in oocytes from 16dpc wild-type (+/+), Top6bl ${ }^{W 562 A / W 562 A}$, and Top $6 b l^{\Delta l 7 C t / \triangle 17 C t}$ ovaries. Scale bar, $10 \mu \mathrm{m}$.

f. Quantification of axis-associated REC114 foci in leptotene and zygotene oocytes from $15 \mathrm{dpc}$ wildtype (+/+), Top6bl ${ }^{W 562 A / W 562 A}$, and Top $6 b l^{\Delta 17 C t / \Delta 17 C t}$ mice (n=1 mouse/genotype). Number of nuclei at leptonema: 51 and 43; number of nuclei at zygonema: 58 and 39 in wild-type and Top6bl ${ }^{\triangle 17 C t / \triangle 17 C t}$ oocytes, respectively. Grey bars show the mean values. P values were determined using the two-tailed unpaired Mann-Whitney test.

Fig. 3 In Top6bl mutants, DSB formation is delayed, DMC1-SSDS signal intensity is decreased in sub-telomeric regions and $X Y$ chromosome synapsis is defective.

a. Immunostaining of $\gamma \mathrm{H} 2 \mathrm{AX}, \mathrm{SYCP} 3$ and SYCP1 in spermatocytes from 12 dpp wild-type $(+/+)$, $T o p 6 b l^{W 562 A / W 562 A}$, and Top $6 b l^{\Delta 17 C t / \triangle 17 C t}$ mice. A white dotted circle highlights the unsynapsed $\mathrm{X}$ and $\mathrm{Y}$ chromosomes at pachynema (SYCP3 staining) in Top $6 b l^{\Delta l 7 C t / \Delta l 7 C t}$ mice. Scale bar, $10 \mu \mathrm{m}$.

b. Quantification of $\gamma \mathrm{H} 2 \mathrm{AX}$ intensity in Top $6 b l^{\mathrm{W562A} / W 562 A}$ and Top $6 b l^{\Delta 17 C t / \Delta 77 C t}$ spermatocytes relative to wild-type at early/mid, late leptotene, and zygotene. Number of nuclei: 19, 201 at early/mid, 33, 290 at late leptotene, and 88, 333 at zygotene in Top $6 b l^{W 562 A / W 562 A}$ and Top $6 b l^{\Delta 17 C t / \Delta 17 C t}$ mice, respectively. The fold reduction is the ratio of the mean values in wild-type and mutant samples. P values were determined using the two-tailed unpaired Mann-Whitney test. 
c. Quantification of DMC1 foci. Axis-associated DMC1 foci were counted in leptotene (early/mid and late), zygotene, and pachytene nuclei of spermatocytes from wild-type (+/+), Top6bl $l^{W 562 A / W 562 A}$, and Top $6 b l^{\Delta 17 C t / \Delta 17 C t}$ mice (n=3 wild-type, and $\mathrm{n}=2$ mice per mutant genotype). Number of nuclei: 95, 50 and 18 at early/mid leptotene, 55, 63 and 45 at late leptotene, 120, 92 and 66 at zygotene, and 261, 207 and 36 at pachytene for each genotype, respectively. Grey bars show the mean values. P values were determined using the two-tailed unpaired Mann-Whitney test.

d. In Top $6 b b^{417 C t / \Delta 17 C t}$ spermatocytes, almost all wild-type hotspots and $20 \%$ of new hotspots are detected. The Venn diagram represents the DMC1-SSDS peaks in wild-type and Top $6 b l^{117 C t / \Delta 17 C t}$ mice. See also Supplementary discussion 3.

e. DMC1-SSDS signal correlation between wild-type and Top $6 b b^{417 C t / \Delta 77 C t}$ mice at wild-type hotspots. The Spearman rho and associated p-value are shown. Ten telomere-proximal hotspots are highlighted for each autosome (blue) and for the X chromosome (red). In the PAR, only part of the DMC1-SSDS signal, which covers a large domain (see panel f), is included within hotspots.

f. DSB maps of the $7 \mathrm{Mb}$ telomere-proximal regions of the chromosomes 19 and $\mathrm{X}$ in wild-type (blue) and Top $6 b l^{\Delta 77 C t / 47 C t}$ (green) mice. PRDM9-independent and -dependent hotspots are identified by orange and blue triangles, respectively. All chromosome ends are shown in Supplementary Fig. 13 and 14.

g. DMC1-SSDS signal intensity decreases in Top $6 b^{417 C t / \Delta 17 C t}$ samples relative to wild-type samples in the q-arm sub-telomeric region. The DMC1-SSDS signal ratio within hotspots (log2-fold change estimated by DESeq2) was computed over $5 \mathrm{Mb}$-windows with a $1 \mathrm{Mb}$-step. Values were averaged for autosomes and plotted separately for the $\mathrm{X}$ chromosome.

h. The q-arm sub-telomeric region effect in Top $6 b l^{\Delta 17 C t / \Delta 17 C t}$ mice. The averaged DMC1-SSDS signal

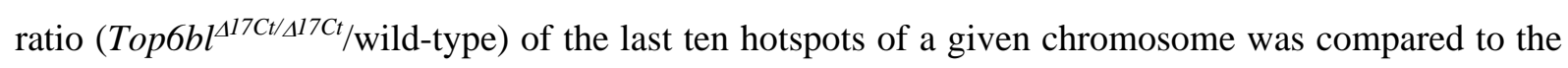
averaged DMC1-SSDS signal ratio of ten randomly chosen, non-telomeric consecutive hotspots in the same chromosome. Boxplots represents the $\log 2$ fold-change (FC) between these values (subtelomeric/non-sub-telomeric) for ten randomizations. The control is shown in Supplementary Fig. 12c i. Synapsis quantification between the $\mathrm{X}$ and $\mathrm{Y}$ chromosomes at pachynema. Synapsis formation was monitored on spreads from late pachytene spermatocytes from adult wild-type $\left(+/+, T_{o p} 6 b^{+/ W 562 A}\right.$ or

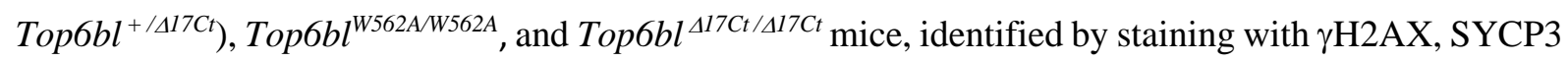
and SYCP1. Number of nuclei: wild-type (227), Top6bl ${ }^{\text {W562A/W562A }}$ (108), Top6bl ${ }^{417 C t / \Delta 17 C t}$ (171). syn: synapsed; unsyn: unsynapsed; UD: undefined. The synapsed/unsynapsed ratios were significantly different between wild-type and Top $6 b l^{W 562 A / W 562 A}$ and Top $6 b l^{\Delta l 7 C t / \Delta l 7 C t}$ spermatocytes (Pearson's ChiSquare, 26.31 and 115.15 , respectively).

j. Quantification of X and Y bivalents at metaphase I. The X and Y chromosomes were detected by FISH in metaphase spreads from adult wild-type $\left(T_{0 p} 6 b l^{+/ \Delta 17 C t}\right)$, Top $6 b l^{\text {W562A/W562A }}$, and Top $6 b l^{\Delta 17 C t / \Delta 17 C t}$ mice ( $\mathrm{n}=2$ mice per genotype). Number of nuclei: 84,144 , and 164 for wild-type, Top $6 b l^{W 562 A / W 562 A}$ and 
Top6bl ${ }^{\triangle 17 C t / \Delta 17 C t}$, respectively. The percentages of metaphase spreads with XY bivalents were significantly different between wild-type and Top $6 b l^{W 562 A / W 562 A}$, and between wild-type and Top $6 b l^{\Delta 17 C t}$ ${ }^{1} \triangle 17 C t$ (Chi-Square Pearson: 54.2 and 96.9, respectively).

k. Representative images of a wild type spermatocyte nucleus with the $\mathrm{X}$ and $\mathrm{Y}$ chromosomes forming a bivalent (left) and of a Top6bl ${ }^{\Delta l 7 C t / \Delta 17 C t}$ spermatocyte nucleus with separated $\mathrm{X}$ and $\mathrm{Y}$ chromosomes (right). Blue, nuclei (DAPI staining); green, X chromosome probe; red, Y chromosome probe. Scale bar, $10 \mu \mathrm{m}$.

\section{Extended Data Fig. 1}

a. The REC114 PH domain (1-159) is sufficient for the interaction with TOPOVIBL. Ni2+ columnpurified TOPOVIBL 450-579 is co-eluted with Strep-REC114 1-159 (PH domain, lane 2), but is not retained by free Strep-Tactin resin (lane 3).

b. Superdex 200 gel filtration elution profiles of REC114 15-159 (blue), TOPOVIBL 450-579 (red), and their complex (green).

c. Disorder prediction of TOPOVIBL analysed by the IUpred2A server (https://iupred2a.elte.hu/). Residues with higher score are more likely to be disordered. While most of the protein is predicted to be rather well structured, the C-terminal part of TOPOVIBL (highlighted in brown) is the most disordered region.

d. Alignment of TOPOVIBL C-terminal regions in metazoans. Helices 5, 6 and 7 in M. musculus TOPOVIBL are part of the transducer domain. Helix 8 is the C-terminal helix. Brown letters: equivalent residues; white letters: identical residues.

e. Superose 6 gel filtration elution profiles of full-length REC114 in complex with MEI4 1-27 (blue), TOPOVIBL 450-579 (red) and their complex (green).

\section{Extended Data Fig. 2}

a. ITC measurement of the interaction affinity between REC114 15-159 and TOPOVIBL 450-579.

b. ITC measurement of the interaction affinity between REC114 15-159 and TOPOVIBL 559-576.

c. REC114-TOPOVIBL structure rotated $60^{\circ}$ around the horizontal axis, compared to Fig. 1f.

d. The central part the TOPOVIBL helix (brown) forms several hydrogen bonds (green dotted lines) with conserved REC114 residues. L569 interacts with another hydrophobic cavity formed by $\beta 6-\beta 8$.

e. The C-terminal W572 residue of TOPOVIBL forms hydrophobic and charged interactions with REC114.

f. His-tag pull-down assays using the proteins shown in Fig. 1i. Strep-tag purified REC114 (WT or mutant) was loaded on $\mathrm{Ni}^{2+}$ columns bound with WT or mutated TOPOVIBL proteins.

g. ITC measurement of the interaction affinity between REC114 15-159 and the TOPOVIBL 559-576 peptide (wild type on the left, and harbouring the W562A mutation on the right). 
h. The key TOPOVIBL and ANKRD31 residues that interact with the REC114 $\beta$-sheet $(\beta 1, \beta 6-\beta 8)$ are in similar positions.

i. ANKRD31-TOPOVIBL competition assay. TOPOVIBL 450-579 was purified on a $\mathrm{Ni}^{2+}$ column (lane 1). Addition of $1 \mathrm{mg}$ of TOPOVIBL 450-579 to Strep-REC114 1-159 is sufficient to form an apparently stoichiometric complex (lane 5). When REC114 is bound to ANKRD31 1808-1857 (lane 6), addition of increasing amount of TOPOVIBL 450-579 (1 to 20mg) is not sufficient to form TOPOVIBL-REC114 complexes (lanes 7-10). Comparison of TOPOVIBL and ANKRD31 binding to REC114 is discussed in Supplementary discussion 1 .

\section{Extended Data Fig. 3}

a. Sequence alignment of the TOPOVIBL proteins from different vertebrate species. Only the sequence of residues 559-576 that include the fragment involved in the interaction with REC114 is shown. Identical residues are in brown boxes. Green squares indicate residues interacting with REC114.

b. The REC114-TOPOVIBL complex models predicted by AlphaFold ${ }^{13}$. The structure of the REC114 PH domain is shown as surface in blue. Key interacting residues of TOPOVIBL are shown as sticks. Most of the interactions observed in the mouse crystal structure are conserved in the predicted complexes of other species. In D. rerio, the residues corresponding to mouse Val566 is Leu555. This mutation seems to be compensated by the valine to glutamine mutation at position 92 in REC114.

\section{Extended Data Fig. 4}

a. Immunostaining of RPA2, SYCP3 and $\gamma \mathrm{H} 2 \mathrm{AX}$ in oocytes from $16 \mathrm{dpc}$ wild-type $(+/+)$, Top $6 b l^{W 562 A / W 562 A}$, and Top $6 b l^{\Delta 17 C t / \Delta 17 C t}$ ovaries. Scale bar, $10 \mu \mathrm{m}$.

b. Quantification of RPA foci per nucleus in leptotene and zygotene oocytes from $16 \mathrm{dpc}$ wild-type (+/+ or Top $6 b l^{+/ \Delta l 7 C t}$ ), Top $6 b l^{W 562 A / W 562 A}$, Top $6 b l^{\Delta 17 C t / \Delta 17 C t}$, and Top $6 b l^{-/-}$mice ( $\mathrm{n}=4$ wild-type and $\mathrm{n}=2$ mice per mutant genotype). Number of nuclei per genotype at leptonema (157, 85, 14, and 29) and at zygonema $(165,55,105$, and 28). Grey bars show the mean value. $P$ values were determined using the two-tailed unpaired Mann-Whitney test. Ratios of the wild-type and mutant mean values are shown.

\section{Extended Data Fig. 5}

a. Immunostaining of ANKRD31, SYCP3 and $\gamma \mathrm{H} 2 \mathrm{AX}$ in oocytes from 16 dpc wild-type (+/+), Top6bl ${ }^{W 562 A / W 562 A}$, and Top $6 b l^{\Delta l 7 C t / \Delta 17 C t}$ ovaries. Scale bar, $10 \mu \mathrm{m}$.

b. Quantification of axis-associated ANKRD31 foci in leptotene and zygotene oocytes from 16 and 18 dpc wild-type (+/+ or Top $\left.6 b l^{+/ \Delta 17 C t}\right), T o p 6 b l^{W 562 A / W 562 A}$, and Top $6 b l^{\Delta 17 C t / \Delta 17 C t}$ mice ( $\mathrm{n}=3$ wild-type, $\mathrm{n}=1$

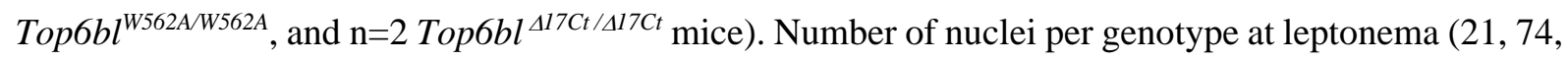
and 56) and at zygonema (56, 57, and 108). Grey bars show the mean values. P values were determined using the two-tailed unpaired Mann-Whitney test. 
c. Immunostaining of MEI4, SYCP3 and $\gamma \mathrm{H} 2 \mathrm{AX}$ in oocytes from $16 \mathrm{dpc}$ wild-type $(+/+)$, Top $6 b l^{W 562 A / W 562 A}$, and Top $6 b l^{\Delta 17 C t / \Delta 17 C t}$ ovaries. Scale bar, $10 \mu \mathrm{m}$.

d. Quantification of axis-associated MEI4 foci in leptotene and zygotene oocytes from 15 and $16 \mathrm{dpc}$ wild-type (+/+ or Top $\left.6 b l^{+/ \Delta 17 C t}\right)$, Top6bl $l^{W 562 A / W 562 A}$, and Top6bl ${ }^{417 C t / \Delta 17 C t}$ mice ( $\mathrm{n}=3$ wild-type, $\mathrm{n}=1$ $T o p 6 b l^{W 562 A / W 562 A}$, and $\mathrm{n}=2 \mathrm{Top}^{\mathrm{W}} 6 \mathrm{l}^{\Delta l 7 C t / \Delta 17 C t}$ mice). Number of nuclei per genotype at leptonema (139, 91, and 42) and at zygonema (106, 24, and 23). Grey bars show the mean values. P values were determined using the two-tailed unpaired Mann-Whitney test.

\section{Extended Data Fig. 6}

a. Immunostaining of $\mathrm{DMC} 1$ and SYCP3 in spermatocytes from $14 \mathrm{dpp}$ wild-type $(+/+)$, Top6bl $l^{W 562 A / W 562 A}$, and Top6bl $l^{\Delta 17 C t / \Delta 17 C t}$ mice. Scale bar, $10 \mu \mathrm{m}$.

b. Quantification of $\gamma \mathrm{H} 2 \mathrm{AX}$ intensity (mean $\pm \mathrm{SD}$; a.u., arbitrary units) in leptotene and zygotene spermatocytes from $12 \mathrm{dpp}$ wild-type, Top $6 b l^{W 562 A / W 562 A}$, and Top $6 b l^{\Delta 17 C t / \Delta 17 C t}$ mice ( $\mathrm{n}=1$ mouse per genotype). Each mutant had a wild-type control performed in parallel. Number of nuclei in the 8 groups plotted on the $\mathrm{x}$ axis: 44, 28, 17, and 34 at leptonema; 132, 61, 77, and 66 at zygonema. $\mathrm{P}$ values were determined using the two-tailed unpaired Mann-Whitney test.

c. Variation of DMC1 focus number during prophase. For each genotype (wild-type, Top $6 b l^{\text {W562A/W562A }}$, and Top $6 b l^{\Delta l 7 C t / \Delta l 7 C t}$ ), the number of DMC1 foci at the indicated stages was normalized to the mean number at zygonema (set to 1). Mean values \pm SD are shown. Statistical significance between wild-type and each mutant (blue Top6bl $l^{W 562 A / W 562 A}$; red Top $6 b l^{\Delta 17 C t / \Delta 17 C t}$ ) was tested at late leptonema using the two-tailed unpaired Mann-Whitney test.

d. Immunostaining of RPA2, SYCP3 and $\gamma \mathrm{H} 2 \mathrm{AX}$ in spermatocytes from 12-14dpp wild-type (+/+), $T o p 6 b l^{W 562 A / W 562 A}$, and Top $6 b l^{\Delta 17 C t / \Delta 17 C t}$ spermatocytes. A white dotted circle highlights the unsynapsed $\mathrm{X}$ and Y chromosomes at pachynema (SYCP3 staining) in Top6bl ${ }^{\Delta l 7 C t / \Delta l 7 C t}$ mice. Scale bar, $10 \mu \mathrm{m}$.

e. Quantification of axis-associated RPA2 foci in early/mid and late leptotene, zygotene and pachytene spermatocytes from 12-14dpp wild-type (+/+ or Top $\left.6 b l^{+/ \Delta 17 C t} ; \mathrm{n}=3\right)$, Top $6 b l^{W 562 A / W 562 A}(\mathrm{n}=2)$, and $T_{o p} 6 b l^{\Delta 17 C t / \Delta 17 C t}(\mathrm{n}=2)$ mice. Number of nuclei per genotype at early/mid leptotene $(105,36$, and 41$)$, late leptotene $(91,38$, and 131), zygotene $(135,62$, and 85), and pachytene $(178,119$, and 80). Grey bars show the mean values. $P$ values were determined using the two-tailed unpaired Mann-Whitney test. f. RPA2 focus variation during prophase. For each genotype (wild-type, Top $6 b l^{W 562 A / W 562 A}$, and Top6bl $\Delta 17 C t / \Delta 17 C t$ ), the number of RPA2 foci at the different stages was normalized to the mean number at zygonema (set to 1). The mean value $\pm \mathrm{SD}$ is shown. Statistical significance between wild-type and each mutant (Top6bl $l^{W 562 A / W 562 A} ;$ Top $6 b l^{\Delta 17 C t / \Delta 17 C t}$ ) was tested at late leptonema using the two-tailed unpaired Mann-Whitney test. 


\section{Extended Data Fig. 7}

a. Immunostaining of REC114 and SYCP3 in spermatocytes from 12-14dpp wild-type (+/+), Top6bl ${ }^{W 562 A / W 562 A}$, and Top $6 b l^{\Delta 17 C t / \Delta 17 C t}$ mice. Scale bar, $10 \mu \mathrm{m}$.

b. Quantification of axis-associated REC114 foci in early/mid and late leptotene, zygotene and pachytene spermatocytes from 12-14dpp wild-type (+/+ or Top $\left.6 b l^{+/ \Delta 17 C t}: \mathrm{n}=4\right)$, Top $6 b l^{W 562 A / W 562 A}(\mathrm{n}=2)$, and Top $6 b l^{\Delta 17 C t / \triangle 17 C t}(\mathrm{n}=2)$ mice. Each mutant is compared with a wild-type control in parallel to control for variations between experiments. Results from Top $6 b l^{W 562 A / W 562 A}$ are from two independent experiments. Number of nuclei: wild-type: 31 early/mid L, 4 late L, 26 Z, 51 P; Top6bl $l^{W 562 A / W 562 A}: 22$ early/mid L, 8 late L, 26 Z, 24 P; wild-type 28 early/mid L, 12 late L, 24 Z, 46 P; Top6bl ${ }^{\text {W562A/W562A }} 18$ early/mid L, 21 late L, 32 Z, 38 P; wild-type: 84 early/mid L, 59 late L, 84 Z, 75 P; Top6bl ${ }^{417 C t / \Delta 17 C t}$ : 76 early/mid L, 68 late L, 79 Z, 61 P. Grey bars show the mean values. P values were determined using the two-tailed unpaired Mann-Whitney test.

c. Variation of REC114 foci during prophase. The number of foci at early/mid leptonema, late leptonema, zygonema and pachynema relative to the mean number at early/mid leptonema was plotted for wild-type, Top $6 b l^{W 562 A / W 562 A}$, and Top $6 b l^{\Delta 17 C t / \Delta 17 C t}$ mice. The mean values \pm SD are shown. Statistical significance between wild-type and each mutant (Top6bl ${ }^{\text {W562A/W562A }}$ and Top6bl ${ }^{417 C t / \Delta 17 C t}$ ) was tested at late leptonema using the two-tailed unpaired Mann-Whitney test.

d. Immunostaining of ANKRD31 and SYCP3 in spermatocytes from 12-14dpp wild-type (+/+), Top $6 b l^{W 562 A / W 562 A}$, and Top6bl ${ }^{\Delta 17 C t / \triangle 17 C t}$ mice. At zygonema, dotted white circles (merge panels) highlight ANKRD31 aggregates at the ends of some chromosome axes. Scale bar, $10 \mu \mathrm{m}$.

e. Quantification of axis-associated ANKRD31 foci in early/mid, late leptotene, zygotene and pachytene spermatocytes from 12-14 dpp wild-type (+/+ or Top $\left.6 b l^{+/ \Delta 17 C t}: \mathrm{n}=2\right), \operatorname{Top} 6 b l^{W 562 A / W 562 A}(\mathrm{n}=1)$, and $\operatorname{Top}_{6 b l^{417 C t / \Delta 17 C t}(\mathrm{n}=1)}$ mice. Mean number of nuclei per genotype at early/mid leptotene $(37,19$, and 23), late leptotene (51, 23, and 19), zygotene (61, 22, and 35), and pachytene (100, 61, and 31). Grey bars show the mean values. P values were determined using the two-tailed unpaired Mann-Whitney test. f. Variation of ANKRD31 foci during prophase. The number of foci at early/mid leptonema, late leptonema, zygonema, and pachynema relative to the mean number at early/mid leptonema (set at 1) was plotted for wild-type, Top $6 b l^{W 562 A / W 562 A}$, and Top $6 b l^{\Delta 17 C t / \Delta 77 C t}$ mice. The mean values \pm SD are shown. Statistical significance between wild-type and each mutant (Top6bl $l^{\text {W562A/W562A }}$ and Top6bl ${ }^{\Delta 17 C t}$ $\Delta \Delta 17 C_{t}$ ) was tested at late leptonema using the two-tailed unpaired Mann-Whitney test.

\section{Extended Data Fig. 8}

a. DMC1-SSDS signal (heatmap and average plots) from wild-type and Top $6 b b^{417 C t / \Delta 17 C t}$ mice at PRDM9 ${ }^{\mathrm{B} 6}{ }_{-}, \mathrm{PRDM}^{\mathrm{RJ} 2}$ - and PRDM9 ${ }^{\mathrm{KO}}$-defined hotspots.

b. MA plot of the DMC1-SSDS signal in Top $6 b l^{\Delta 17 C t / \Delta 77 C t}$ compared with wild-type samples. The $\log 2$ of the ratios was calculated using DESeq2 for differential analysis and after log fold change shrinkage. 
Hotspot with significantly increased or decreased signal are highlighted in red $(n=214)$ and green ( $\mathrm{n}=1098$ ), respectively (adjusted $\mathrm{p}$-value $<0.1)$. Unchanged hotspots are in grey $(\mathrm{n}=8043)$. The mean normalized count corresponds to the baseMean value from the DESeq2 analysis.

c. Decreased (green), increased (red), or unchanged (grey) hotspot density within the $3 \mathrm{Mb}$ sub-telomeric (Subtelo) region relative to the non-sub-telomeric regions (NonSubtelo). Decreased, increased and unchanged hotspots were determined from the DESeq2 analysis, as shown in panel $b$. The densities of decreased and unchanged hotspots in the two studied regions were compared using the Pearson's Chisquare test, and p-values were adjusted for multiple testing using the Benjamini \& Yekutieli method. Yellow stars indicate $\mathrm{p}$-value $<0.05$. The Chi-square test results are provided in Supplementary Table S2.

d. Sub-telomeric/Non-sub-telomeric hotspot density ratios for unchanged, decreased, and increased hotspots, as defined by DESeq2 analysis, for sub-telomeric regions defined using various distances from the telomeric annotated ends (1-2-3-4-5-7-10Mb). The $\log 2$ ratio was plotted and showed excess (positive values) or lack (negative values) of hotspots at sub-telomeric regions compared with non-subtelomeric regions.

\section{Extended Data Fig. 9}

a. Quantification of homologous synapses in Top $6 b l$ mutants. The percentage of pachytene nuclei with 19 fully synapsed autosomes was determined by SYCP3 and SYCP1 staining of adult spermatocytes from wild-type (+/+, Top $6 b l^{+/ W 562 A}$ or Top $\left.6 b l^{+/ \Delta 17 C t}\right), T_{o p} 6 b l^{W 562 A / W 562 A}$, and Top $6 b l^{\Delta 17 C t / \Delta 17 C t}$ mice.

b. Representative images of DAPI-stained wild-type and Top $6 b l^{\Delta 17 C t / \Delta 17 C t}$ metaphase spreads. Scale bar, $10 \mu \mathrm{m}$. DAPI-stained bodies are numbered (arbitrarily): 20 are observed in wild-type, and 21 in Top6bl ${ }^{\Delta 17 C t / \Delta 17 C t}$ metaphase spreads.

c. Quantification of bivalent formation at metaphase I. Percentage of metaphases with 20, 21, 20 or 21, or $>21$ DAPI-stained bodies per nucleus from adult wild-type $\left(T o p 6 b l^{+/ \Delta 17 C t}\right), T o p 6 b l^{W 562 A / W 562 A}$, and Top $6 b l^{\Delta 17 C t / \triangle 17 C t}$ mice ( $\mathrm{n}=2$ mice per genotype). Number of nuclei: 75,80 , and 97 , respectively. The number of nuclei with 20 and 21 bivalents was significantly different between wild-type and Top6bl ${ }^{W 562 A / W 562 A}$ and between wild-type and Top $6 b l^{\Delta 17 C t / \Delta 77 C t}$ metaphases (Pearson's Chi-Square: 44.4 and 78.8 respectively).

\section{Extended Data Fig. 10}

a. The structure of the complex formed by SPO11 and the C-terminal part of the TOPOVIBL transducer domain was modelled with AlphaFold ${ }^{13}$. The full-length TOPOVIBL modelled structure (AF-J3QMY9F1) was superimposed on the transducer domain. The structure of REC114 PH domain bound to TOPOVIBL C-terminus was determined in this study and is shown linked to the SPO11-TOPOVIBL 
complex via a long-disordered linker. No contact with the SPO11-TOPOVIBL complex could be modelled.

b. According to the AlphaFold model, the putative ATP binding site of TOPOVIBL is degenerated. Secondary structure elements forming the ATP binding site in S. shibatae (PDB - 1MXO, lower panel), are organized differently in mouse TOPOVIBL. Consequently, a corresponding ATP binding site is not formed. Mouse TOPOVIBL also lacks the N-terminal "strap" region and the H2TH domain, both involved in TopoVIB dimerization, indicating that TOPOVIBL dimerization, if occurs, should differ from that of TopoVIB ${ }^{47}$.

c. The modelled SPO11 dimerization interface resembles the one described for archaeal TopoVIA (bottom), including the formation of a pseudo-continuous $\beta$-sheet by the two protomers ${ }^{48}$.

900 d. The modelled SPO11 catalytic site is equivalent to the one previously described for TopoVIA (PDB - 1D3Y, lower panel) where the two protomers contribute three negatively charged residues that coordinate the magnesium atom and the catalytic tyrosine ${ }^{48}$.

e. The modelled SPO11-TOPOVIBL interface is similar to that of the archaeal TopoVI complex (PDB -2Q2E, lower panel), with the additional helix of the TOPOVIBL transducer domain ${ }^{2,3}$. 
bioRxiv preprint doi: https://doi.org/10.1101/2021.11.30.470517; this version posted December 2,2021 . The copyright holder for this preprint (which was not certified by peer review) is the author/funder, who has granted bioRxiv a license to display the preprint in perpetuity. It is made available under aCC-BY-NC-ND 4.0 International license.

\section{References}

1 Bergerat, A. et al. An atypical topoisomerase II from Archaea with implications for meiotic 910 recombination [see comments]. Nature 386, 414-417 (1997).

2 Corbett, K. D., Benedetti, P. \& Berger, J. M. Holoenzyme assembly and ATP-mediated conformational dynamics of topoisomerase VI. Nat Struct Mol Biol 14, 611-619 (2007).

3 Graille, M. et al. Crystal Structure of an Intact Type II DNA Topoisomerase: Insights into DNA Transfer Mechanisms. Structure 16, 360-370 (2008).

$4 \quad$ Keeney, S., Giroux, C. N. \& Kleckner, N. Meiosis-specific DNA double-strand breaks are catalyzed by Spo11, a member of a widely conserved protein family. Cell 88, 375-384 (1997). Robert, T. et al. The TopoVIB-Like protein family is required for meiotic DNA double-strand break formation. Science 351, 943-949 (2016).

6 Vrielynck, N. et al. A DNA topoisomerase VI-like complex initiates meiotic recombination. Science 351, 939-943 (2016).

7 Grey, C., Baudat, F. \& de Massy, B. PRDM9, a driver of the genetic map. PLoS Genet 14, e1007479 (2018).

8 Imai, Y. et al. PRDM9 activity depends on HELLS and promotes local 5-hydroxymethylcytosine enrichment. eLife 9 (2020).

9 Spruce, C. et al. HELLS and PRDM9 form a pioneer complex to open chromatin at meiotic recombination hot spots. Genes Dev 34, 398-412 (2020).

10 Boekhout, M. et al. REC114 Partner ANKRD31 Controls Number, Timing, and Location of Meiotic DNA Breaks. Mol Cell 74, 1053-1068 e1058 (2019).

11 Kumar, R. et al. Mouse REC114 is essential for meiotic DNA double-strand break formation and forms a complex with MEI4. Life science alliance 1, e201800259 (2018).

12 Papanikos, F. et al. Mouse ANKRD31 Regulates Spatiotemporal Patterning of Meiotic Recombination Initiation and Ensures Recombination between $X$ and $Y$ Sex Chromosomes. Mol Cell 74, 1069-1085 e1011 (2019).

13 Jumper, J. et al. Highly accurate protein structure prediction with AlphaFold. Nature 596, 583-589 (2021).

14 Cohen, P. E., Pollack, S. E. \& Pollard, J. W. Genetic analysis of chromosome pairing, recombination and cell cycle control during first meiotic prophase in mammals. Endocr Rev 27, 398-426. (2006).

15 Mahadevaiah, S. K. et al. Recombinational DNA double-strand breaks in mice precede synapsis. Nat Genet 27, 271-276. (2001).

16 Baudat, F., Imai, Y. \& de Massy, B. Meiotic recombination in mammals: localization and regulation. Nat Rev Genet 14, 794-806 (2013).

17 Hinch, A. G. et al. The Configuration of RPA, RAD51, and DMC1 Binding in Meiosis Reveals the Nature of Critical Recombination Intermediates. Mol Cell 79, 689-701 e610 (2020).

18 Fraune, J., Schramm, S., Alsheimer, M. \& Benavente, R. The mammalian synaptonemal complex: protein components, assembly and role in meiotic recombination. Exp Cell Res 318, 1340-1346 (2012).

19 Kumar, R., Bourbon, H. M. \& de Massy, B. Functional conservation of Mei4 for meiotic DNA double-strand break formation from yeasts to mice. Genes Dev 24, 1266-1280 (2010).

95020 Libby, B. J., Reinholdt, L. G. \& Schimenti, J. C. Positional cloning and characterization of Mei1, a vertebrate-specific gene required for normal meiotic chromosome synapsis in mice. Proc Natl Acad Sci U S A 100, 15706-15711 (2003).

21 Stanzione, M. et al. Meiotic DNA break formation requires the unsynapsed chromosome axisbinding protein IHO1 (CCDC36) in mice. Nat Cell Biol 18, 1208-1220 (2016).

22 Kumar, R. et al. MEI4 - a central player in the regulation of meiotic DNA double-strand break formation in the mouse. J Cell Sci 128, 1800-1811 (2015). 
bioRxiv preprint doi: https://doi.org/10.1101/2021.11.30.470517; this version posted December 2,2021 . The copyright holder for this preprint (which was not certified by peer review) is the author/funder, who has granted bioRxiv a license to display the preprint in perpetuity. It is made available under aCC-BY-NC-ND 4.0 International license.

23 Khil, P. P., Smagulova, F., Brick, K. M., Camerini-Otero, R. D. \& Petukhova, G. V. Sensitive mapping of recombination hotspots using sequencing-based detection of ssDNA. Genome Res 22, 957-965 (2012).

96024 Brick, K., Smagulova, F., Khil, P., Camerini-Otero, R. D. \& Petukhova, G. V. Genetic recombination is directed away from functional genomic elements in mice. Nature 485, 642645 (2012).

25 Odorisio, T., Rodriguez, T. A., Evans, E. P., Clarke, A. R. \& Burgoyne, P. S. The meiotic checkpoint monitoring synapsis eliminates spermatocytes via p53-independent apoptosis. Nat Genet 18, 257-261 (1998).

26 Kauppi, L. et al. Distinct Properties of the XY Pseudoautosomal Region Crucial for Male Meiosis. Science 331, 916-920 (2011).

27 Claeys Bouuaert, C. et al. DNA-driven condensation assembles the meiotic DNA break machinery. Nature 592, 144-149 (2021).

$97028 \quad$ Vrielynck, N. et al. Conservation and divergence of meiotic DNA double strand break forming mechanisms in Arabidopsis thaliana. Nucleic Acids Res 49, 9821-9835 (2021).

29 Scherthan, H. Telomere attachment and clustering during meiosis. Cell Mol Life Sci 64, 117124 (2007).

30 Shibuya, H. et al. MAJIN Links Telomeric DNA to the Nuclear Membrane by Exchanging Telomere Cap. Cell 163, 1252-1266 (2015).

31 Smagulova, F. et al. Suppression of genetic recombination in the pseudoautosomal region and at subtelomeres in mice with a hypomorphic Spo11 allele. BMC Genomics 14, 493 (2013).

32 Yamada, S. et al. Genomic and chromatin features shaping meiotic double-strand break formation and repair in mice. Cell Cycle, 0 (2017).

561, 338-342 (2018).

34 Gruhn, J. R., Rubio, C., Broman, K. W., Hunt, P. A. \& Hassold, T. Cytological studies of human meiosis: sex-specific differences in recombination originate at, or prior to, establishment of double-strand breaks. PLoS One 8, e85075 (2013).

35 Peters, A. H., Plug, A. W., van Vugt, M. J. \& de Boer, P. A drying-down technique for the spreading of mammalian meiocytes from the male and female germline. Chromosome Res 5 , 66-68. (1997).

36 Grey, C., Baudat, F. \& de Massy, B. Genome-Wide Control of the Distribution of Meiotic Recombination. PLoS Biol 7, e35 (2009).

37 Grey, C. et al. In vivo binding of PRDM9 reveals interactions with noncanonical genomic sites. Genome Res 27, 580-590 (2017).

38 Landt, S. G. et al. ChIP-seq guidelines and practices of the ENCODE and modENCODE consortia. Genome Res 22, 1813-1831 (2012).

39 Love, M. I., Huber, W. \& Anders, S. Moderated estimation of fold change and dispersion for RNA-seq data with DESeq2. Genome Biol 15, 550 (2014).

40 Zhu, A., Ibrahim, J. G. \& Love, M. I. Heavy-tailed prior distributions for sequence count data: removing the noise and preserving large differences. Bioinformatics 35, 2084-2092 (2019).

41 Kabsch, W. Xds. Acta crystallographica. Section D, Biological crystallography 66, 125-132

42 McCoy, A. J. et al. Phaser crystallographic software. Journal of applied crystallography 40, 658-674 (2007).

43 Terwilliger, T. C. Maximum-likelihood density modification. Acta crystallographica. Section D, Biological crystallography 56, 965-972 (2000).

44 Emsley, P., Lohkamp, B., Scott, W. G. \& Cowtan, K. Features and development of Coot. Acta crystallographica. Section D, Biological crystallography 66, 486-501 (2010). 
bioRxiv preprint doi: https://doi org/10.1101/2021.11.30.470517; this version posted December 2,2021 . The copyright holder for this

45 Murshudov, G. N., Vagin, A. A. \& Dodson, E. J. Refinement of macromolecular structures by the maximum-likelihood method. Acta crystallographica. Section D, Biological crystallography 53, 240-255 (1997).

46 Chen, V. B. et al. MolProbity: all-atom structure validation for macromolecular crystallography. Acta crystallographica. Section D, Biological crystallography 66, 12-21 (2010).

47 Corbett, K. D. \& Berger, J. M. Structure of the topoisomerase VI-B subunit: implications for type II topoisomerase mechanism and evolution. EMBO J. 22, 151-163 (2003).

48 Nichols, M. D., DeAngelis, K., Keck, J. L. \& Berger, J. M. Structure and function of an archaeal topoisomerase VI subunit with homology to the meiotic recombination factor Spo11. Embo J 18, 6177-6188 (1999).

49 Jones, D. T. Protein secondary structure prediction based on position-specific scoring matrices. J Mol Biol 292, 195-202 (1999).

Fedorov, A. A., Fedorov, E., Gertler, F. \& Almo, S. C. Structure of EVH1, a novel proline-rich ligand-binding module involved in cytoskeletal dynamics and neural function. Nature structural biology 6, 661-665 (1999). 
a

REC114

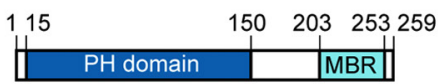

b

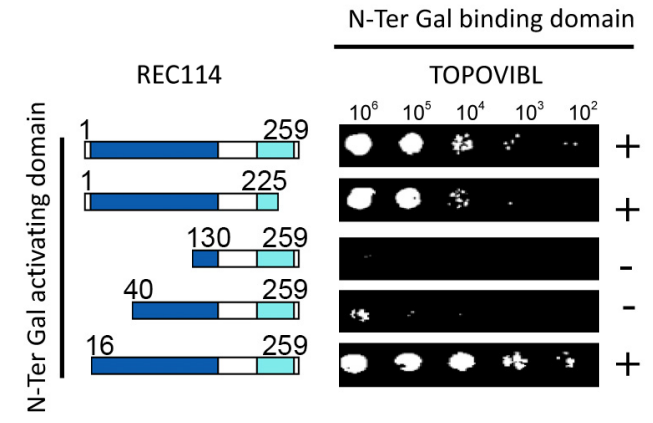

d

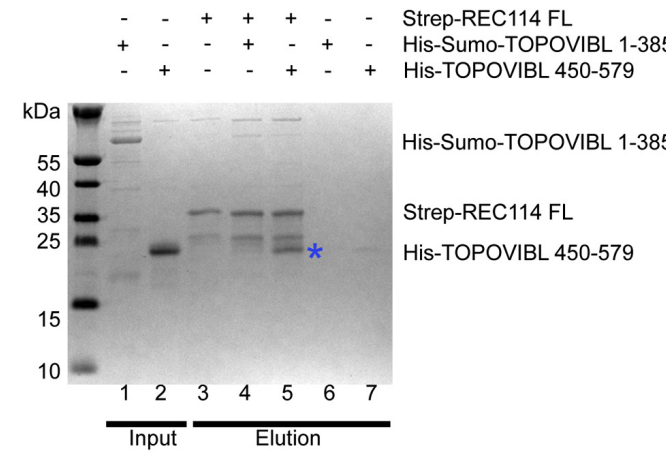

9

$\mathbf{f}$

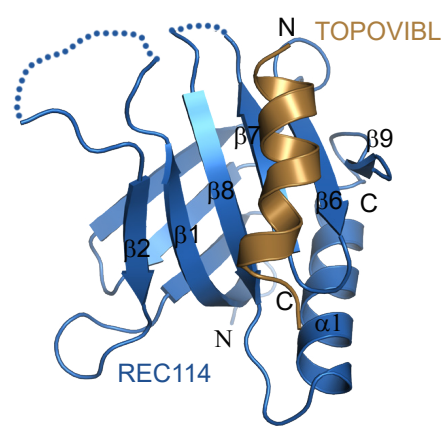

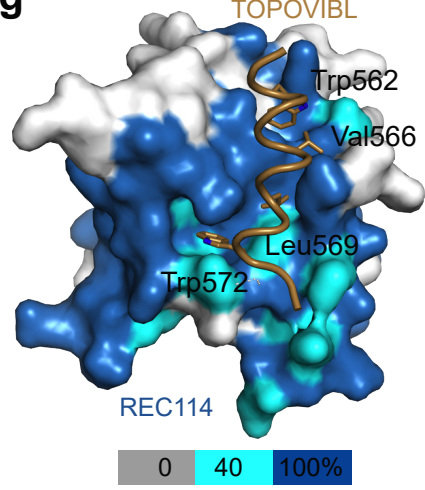

h

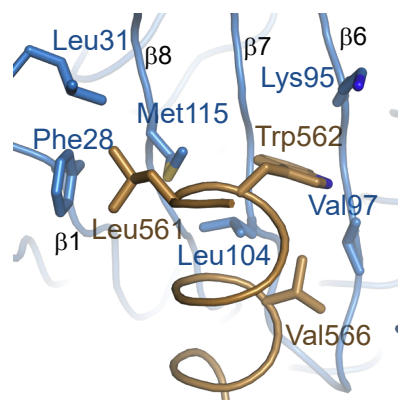

Mus musculus

Homōsapiens

Divittatum 196 DKLWLEVSKLSD

Chelonia mydas

Latimeria_chalumnae 619 DDWWLQEVIRL SEW

Crassostréa gigas 708 MDAKLDEALLEMKD

Patiria miniata 189 EDEWLDEVLDSDP

Nematostella_vectensis 301 SFDWLEEFENNTFW

Strep-Tactin resin
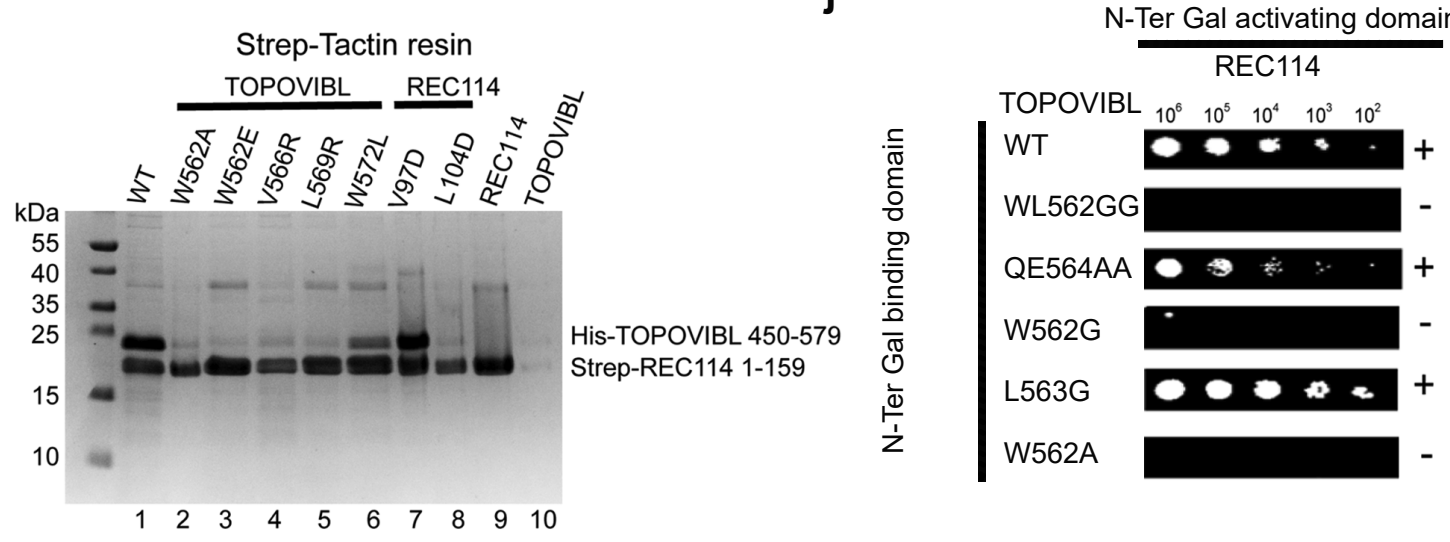
a

wild-type

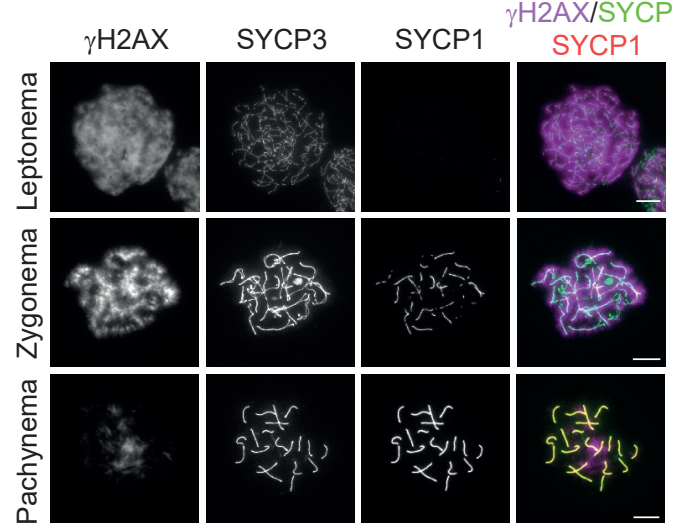

Top6b/W652A/W562A

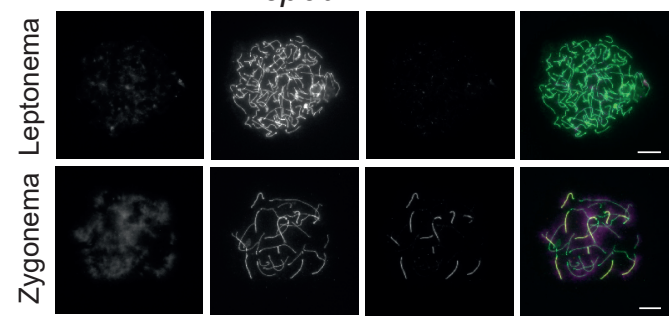

Top6b/17Ct/A17Ct

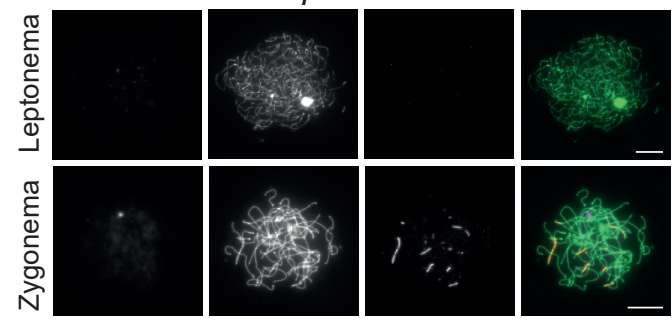

C

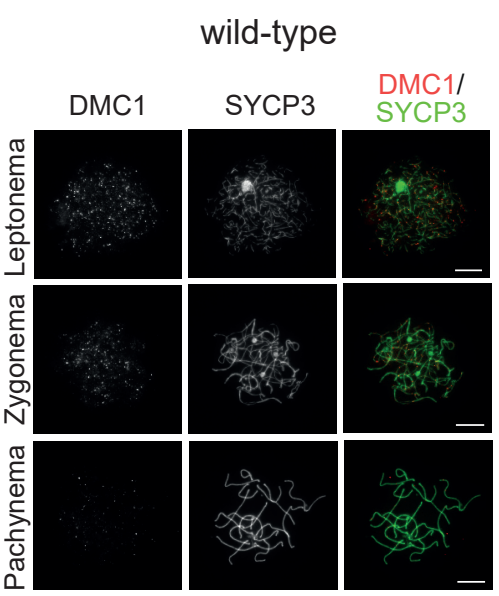

Top6b/W652A/W562A

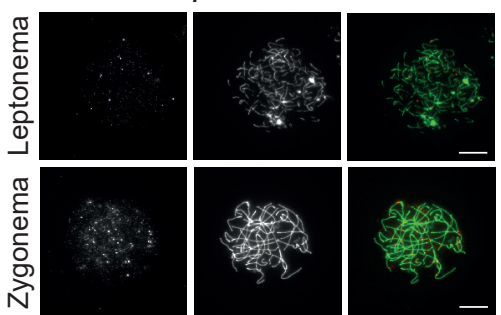

$T o p 6 b^{117 C t / \Delta 17 c t}$

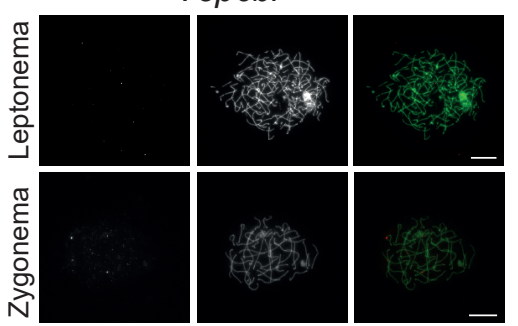

e

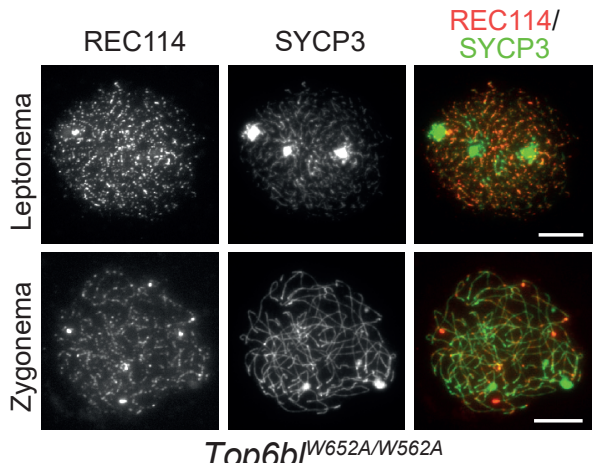

Top6b/W652A/W562A

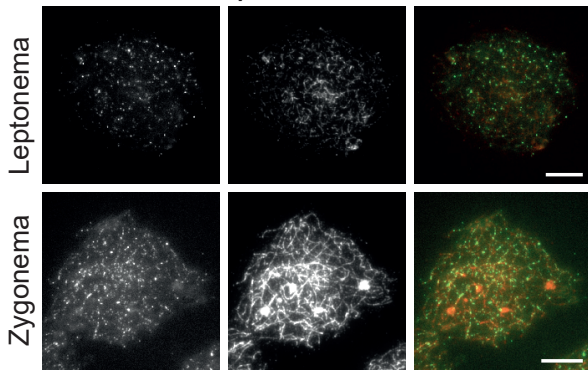

Top $6 b^{\Delta 17 c t / \Delta 17 c t}$
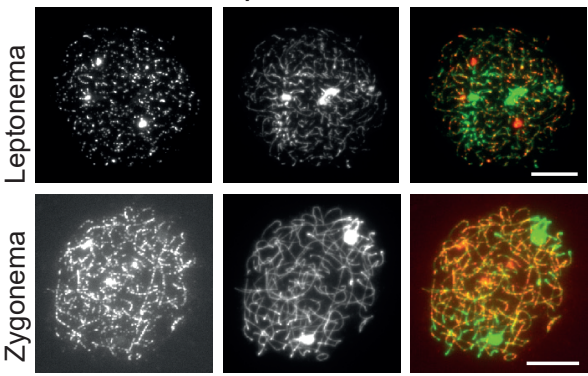

b

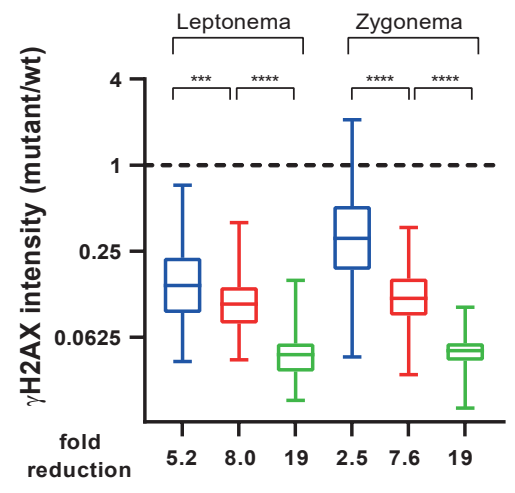

d

f
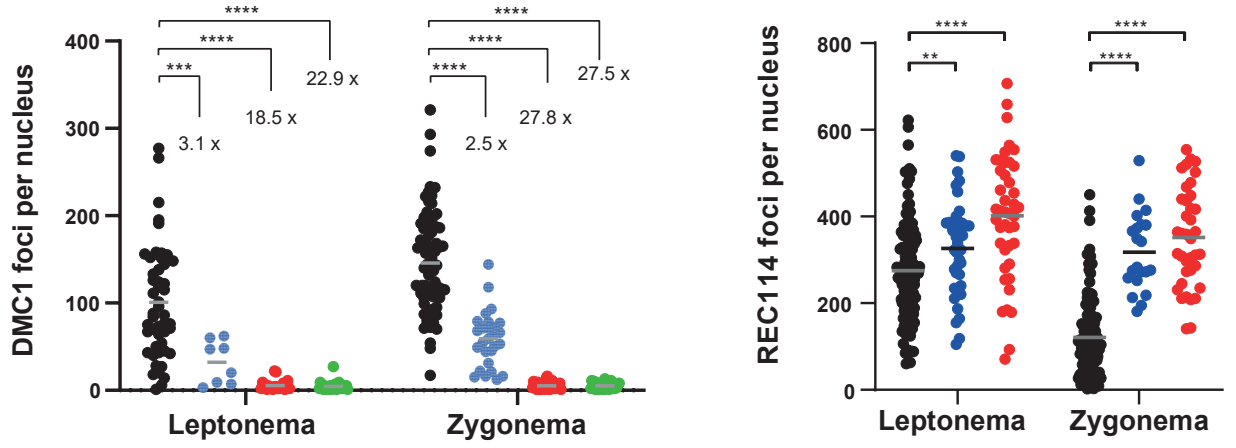

- wild type „Top6bl ${ }^{\text {W562AW562A }}$ - Top6bl ${ }^{417 C t / \Delta 17 C t}$.Top6bl ${ }^{-1}$ 
a $\quad$ bioRxiv preprint doi: https://doi.org/10.1101/2021.11.30.470517; this version posted December 2, 2021. The copyright holder for this Figure 3
preprint (which was not certified by peer review) is the authorffunder who has granted bioRxiv a license to display the preprint in

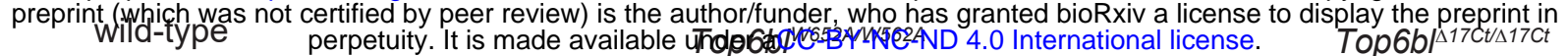

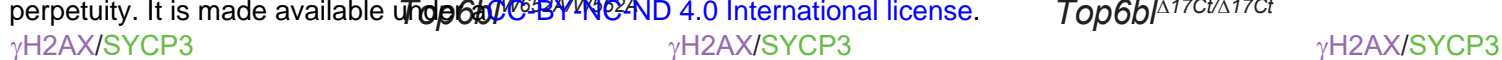

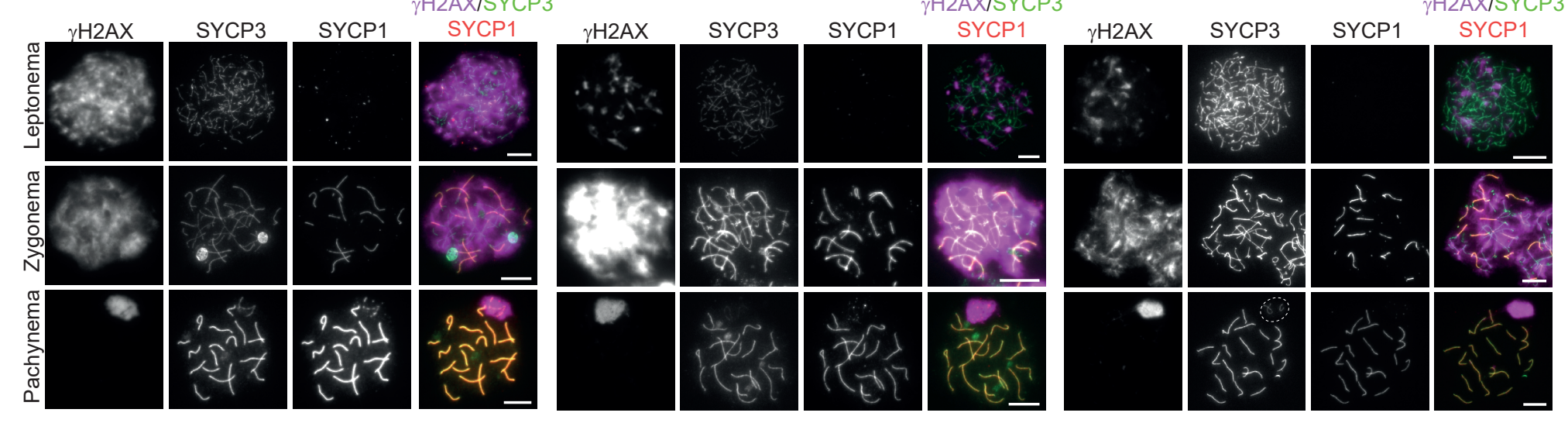

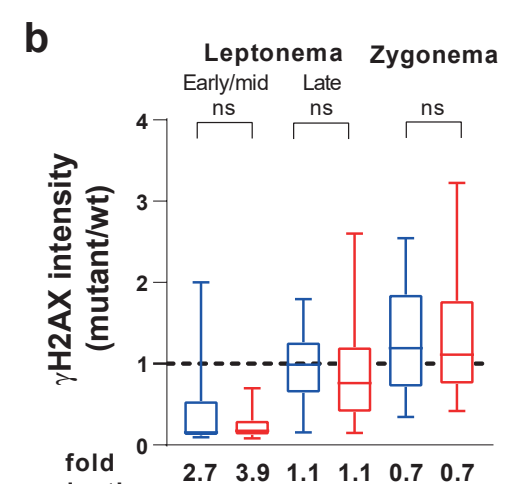

- wild type "Top6b/ ${ }^{\text {W562Aw562A }}$. Top6b/ ${ }^{417 C t / \Delta 17 C t}$

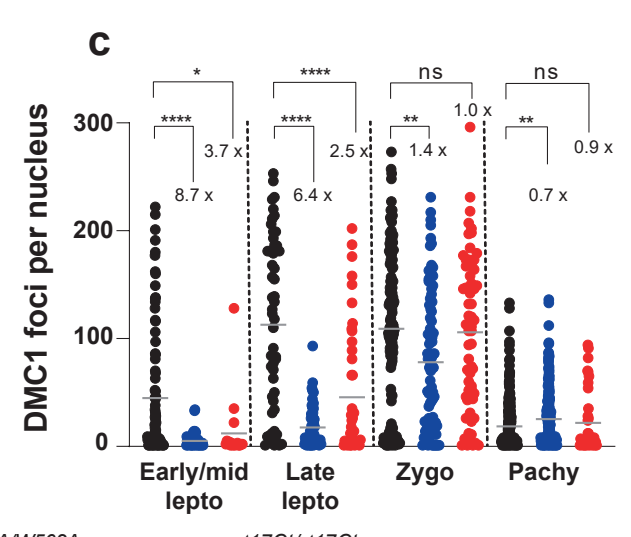

d

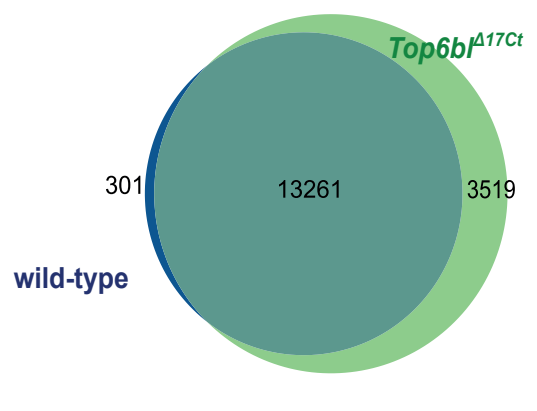

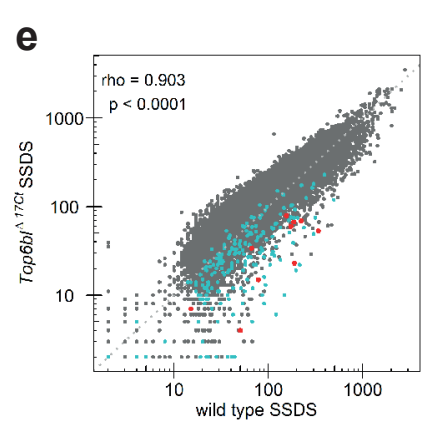

f chr19- last 7Mb

wild-type

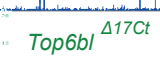

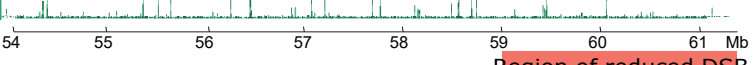

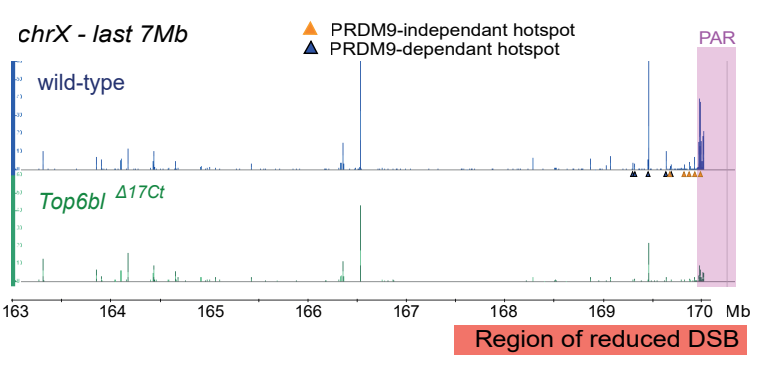

h
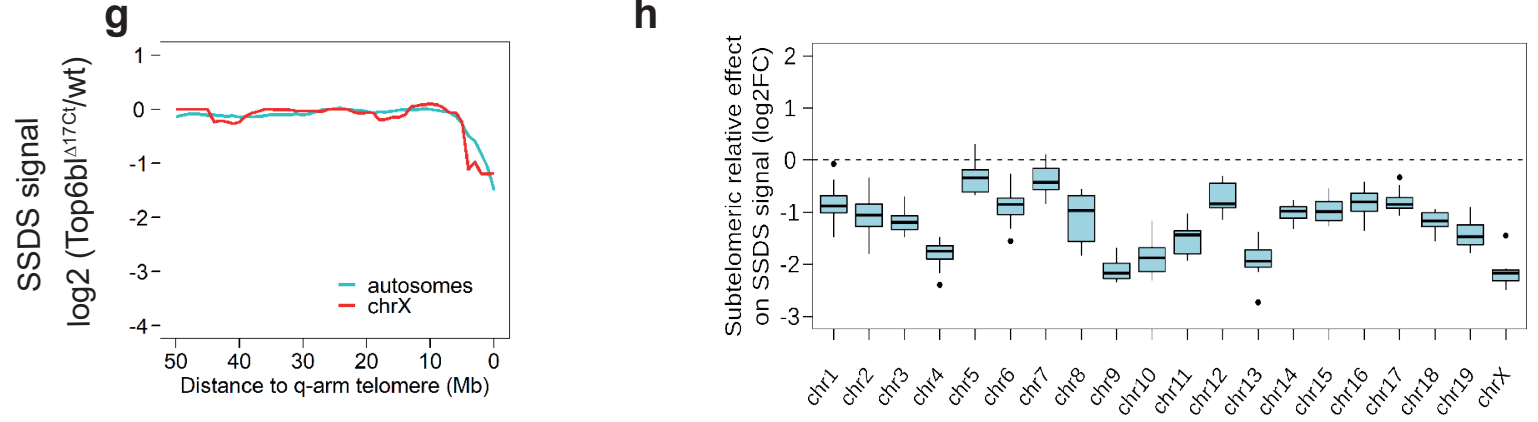

Chromosome

k
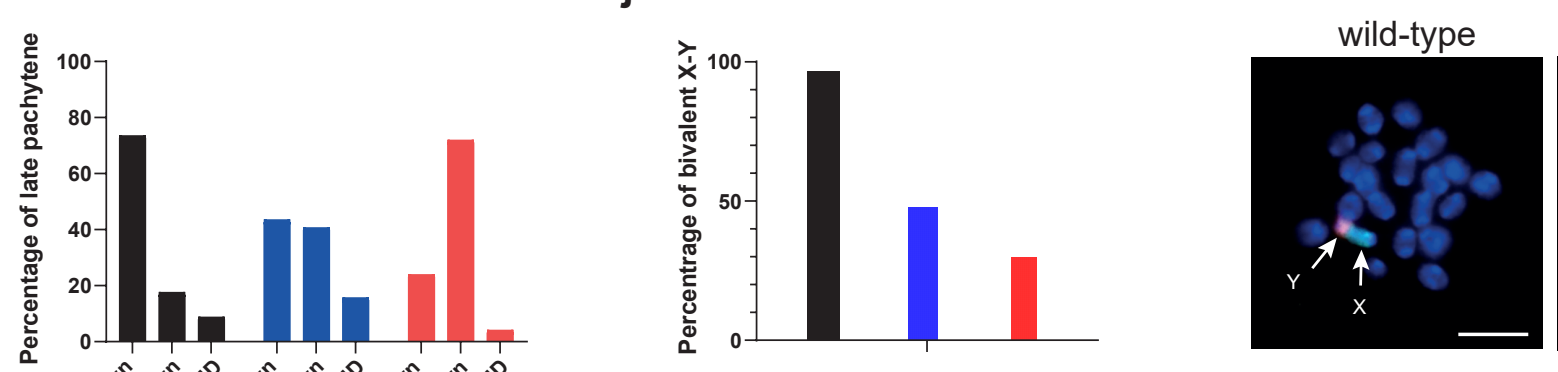

Top6b/17ct/17Ct

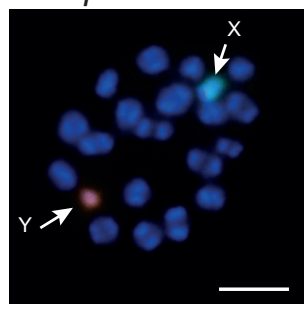

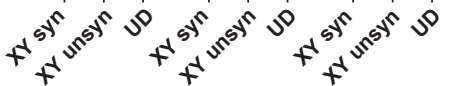

- wild type "Top6b/ ${ }^{\text {W562Aw562A }}$. Top6b/ ${ }^{417 C t / 117 C t}$ 
a

Strep-REC114 1-159 + + -

His-TOPOVIBL 450-579 $\quad-\quad+\quad+$

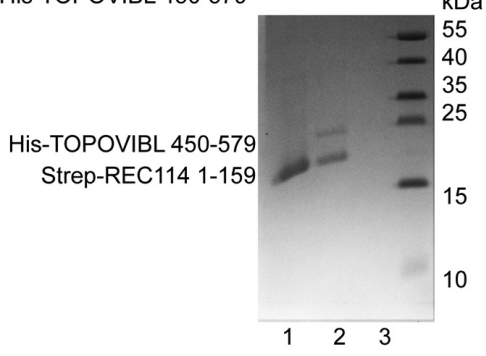

C

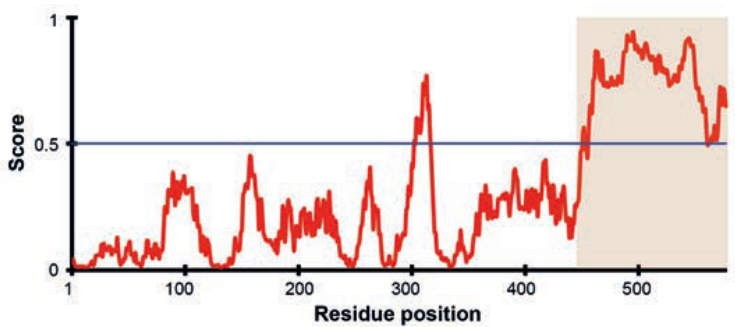

b

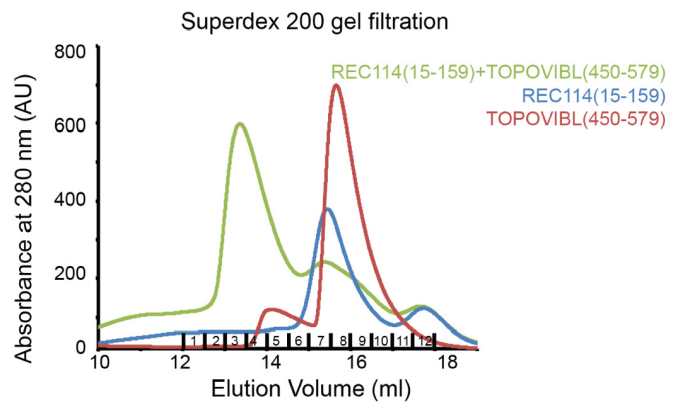

Superose 6 gel filtration

d
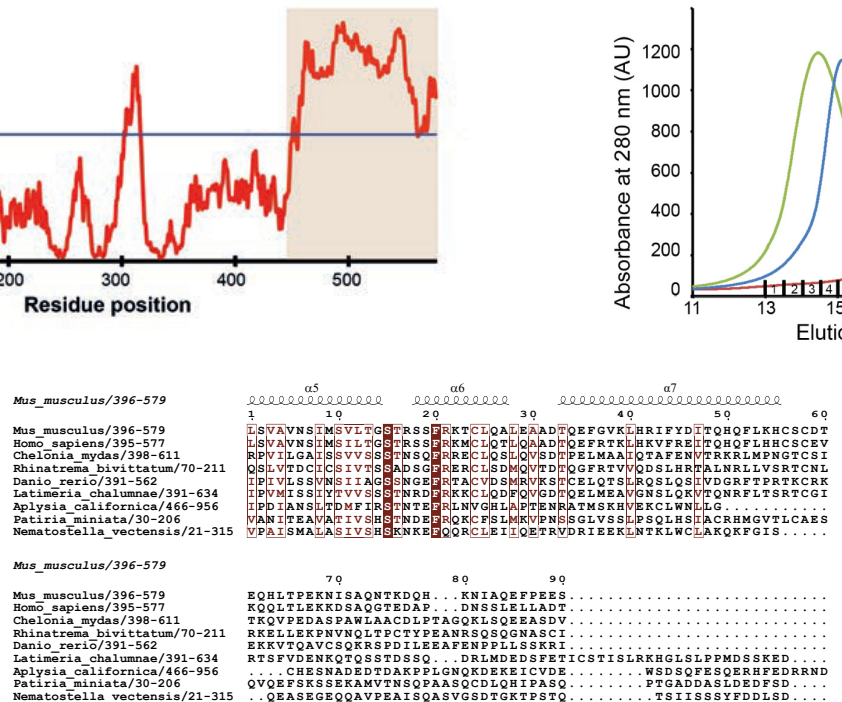

REC114+MEI4(1-127)+TOPOVIBL(450-579) REC114+MEI4(1-127)

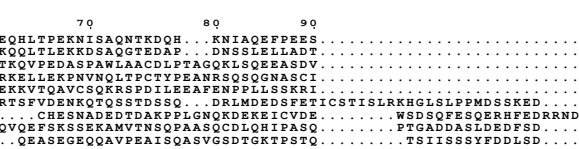

Mus_musculus/396-579 Mus musculus $/ 396-579$
Homo- sapiens $395-577$
Chelonia mydas $/ 398-611$ Rhinatrema-bivittatum/70-211 Danio-rerio/ $391-562$
Latimeria_chalumae/391-634
Aplysia_cálifornica/466-956 Patiria -miniata/30-206-956
Nematostella vectensis/21-31

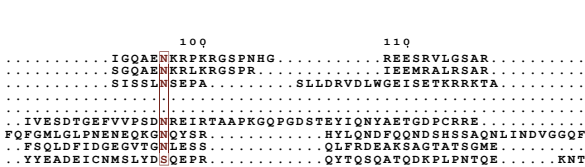
Mus_musculus/396-579 Mus musculus $/ 396-579$
Homo_sapiens/395-577 Chelonia_mydas/398-611
Rhinatrema bivittatum/70-211 Danio-rerio/ $391-562$ (3)

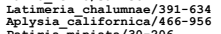

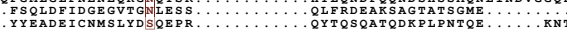
Mus_musculus/396-579 Mus_musculus $/ 396-579$
Homo_sapiens/395-577 Chelonia mydas $/ 398-611$
Rhinatrema bivittatum/70-211

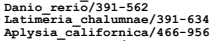
Patiria-miniata/30-206
Nematostella_vectensis/21-315

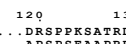
130 .

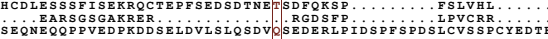
Mus_musculus/396-579 Mus musculus/396-579 Cheiōnia mydas $/ 398-611$ Rhinatrema_bivittatum/70-21
Danio-rerio $391-562$
Latimeria_chalumnae/391-634 Aplysia_cálifornica/466-956 TLQNDHKETTLRQTNVADQSIIEISDSEDSPVTKAKNVPLEPHVSIIKTCKLDQPAHE Mus_musculus/396-579

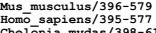
Rhinatrema bivittatum/70-211 Danio-rerio/ $391-562$
Latimeria-chalumnae/391-634 APlysia_câlifornica/466-956 MSDRDQCTEPLQSEQEKNDEHCETPKSLSLVSVVCTDFEKRPCFPRKRSSEYI Mus_musculus/396-579 Mus musculus $/ 396-579$
Homó_sapiens $/ 395-577$ Chelonia-mydas/398-611
Rhinatrema-bivittatum/70-211 Latimeria_chalumnae/391-634 Aatiria-miniata/30-2066-95

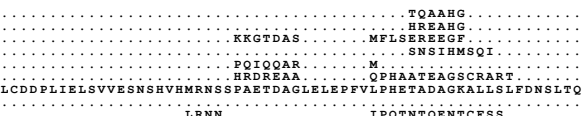
.................... IPQTNTQENTCFSS

Mus_musculus/396-579 Mus_musculus $/ 396-579$
Homo-sapiens/395-577 
a

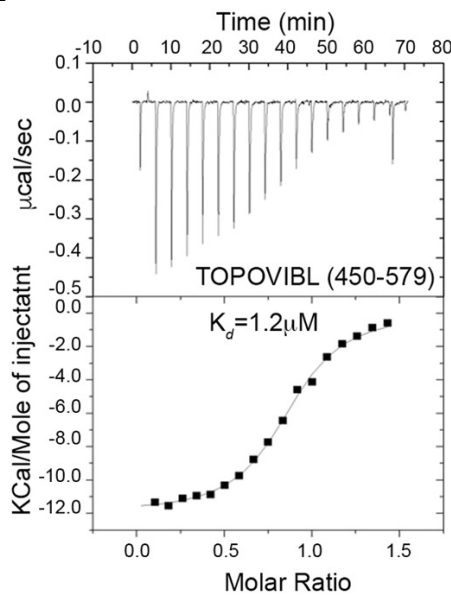

d

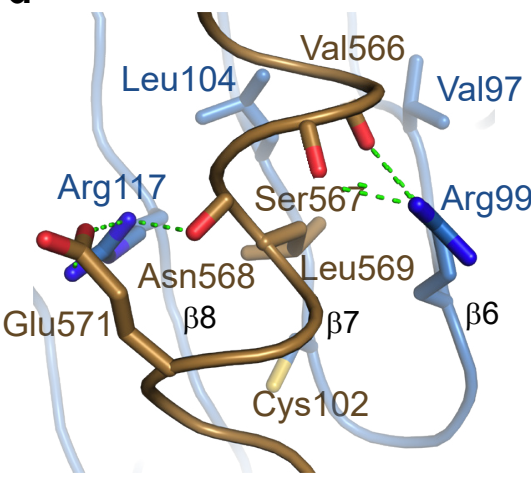

f

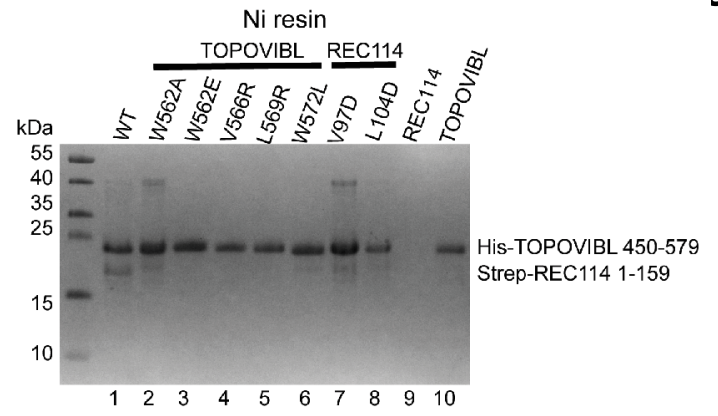

h

b

e

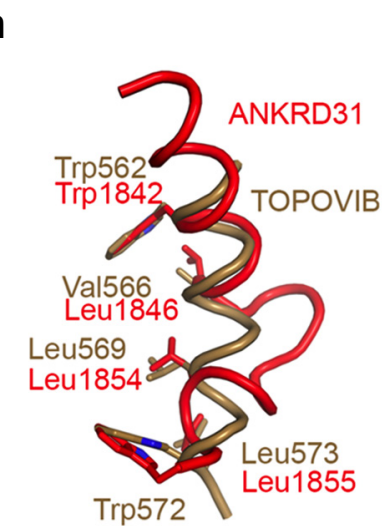

Trp1857

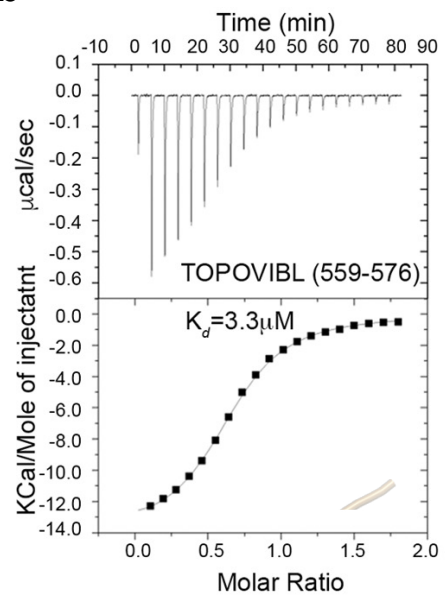

C

Extended Data Fig.2

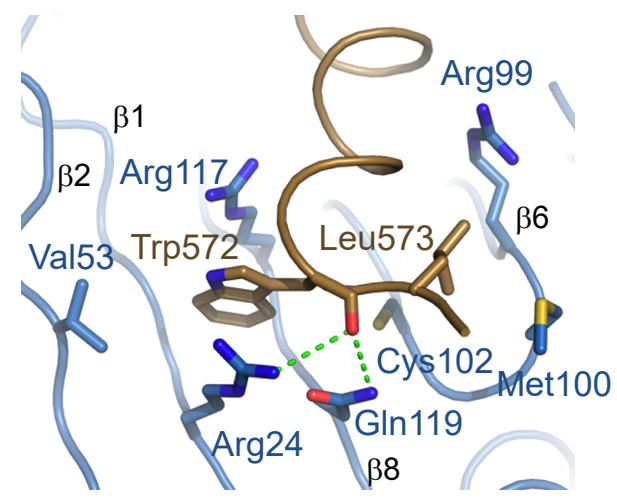

g
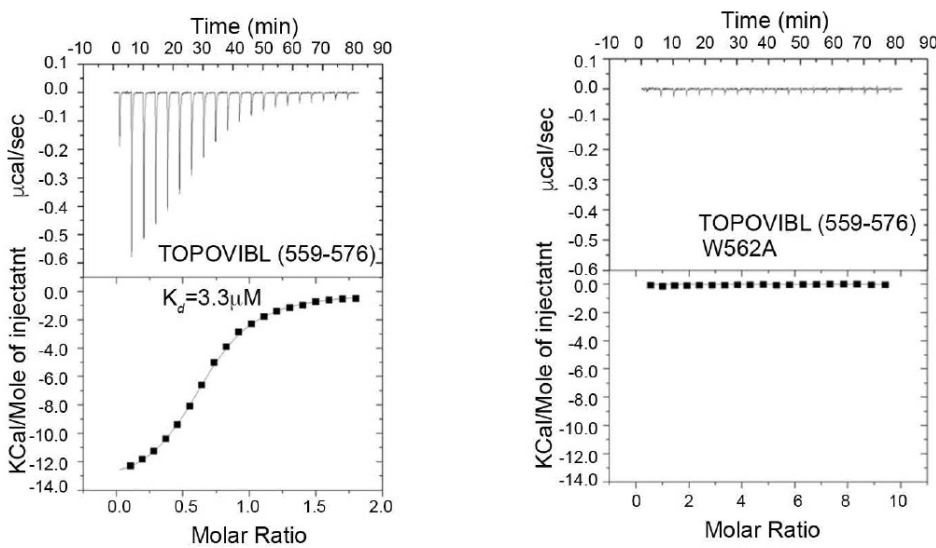

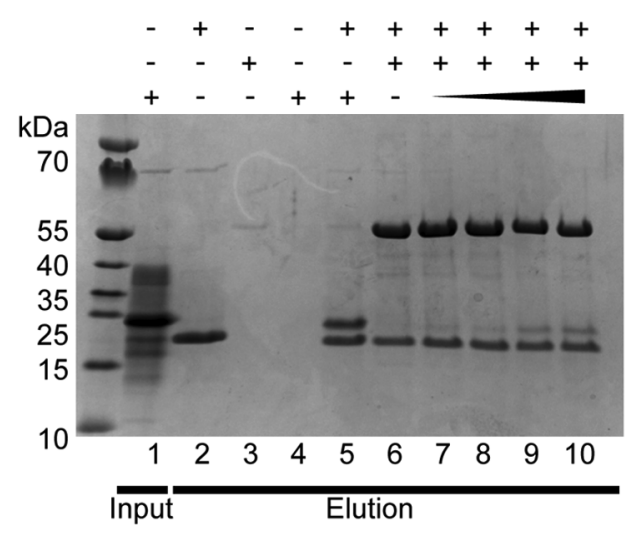

Strep-REC114 1-159

His-MBP-ANKRD31 1808-1857

His-TOPOVIBL 450-579

His-MBP-ANKRD31 1808-1857

His-TOPOVIBL 450-579

Strep-REC114 1-159 
bioRxiv preprint doi: https://doi.org/10.1101/2021.11.30.470517; this version posted December 2, 2021. The copyright holder for this preprint (which was not certified by peer review) is the author/funder, who has granted bioRxiv a license to display the preprint in

a perpetuity. It is made available under aCC-BY-NC-ND 4.0 International license.

\section{b}

\begin{tabular}{|c|c|}
\hline \multirow{8}{*}{$\begin{array}{l}\text { Mus_musculus } \\
\text { Homo_sapiens } \\
\text { Phoca_vitulina } \\
\text { Ornithorhynchus_anatinus } \\
\text { Chelonia_mydas } \\
\text { Rhinatrema_bivittatum } \\
\text { Danio_rerio } \\
\text { Latimeria_chalumnae }\end{array}$} & 559 EDLWLOEVSNISEWLNP \\
\hline & 557 DVL WLQ EV S N L SEWL SP \\
\hline & 509 DSLWQQEV SNLSEWL SP \\
\hline & 528 ELLQ \\
\hline & 596 DHLWLQEISNLSEWTS. \\
\hline & 196 DKLWLQEVSKL \\
\hline & QQWI ELENESEWD . \\
\hline & 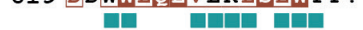 \\
\hline
\end{tabular}

Homo sapiens

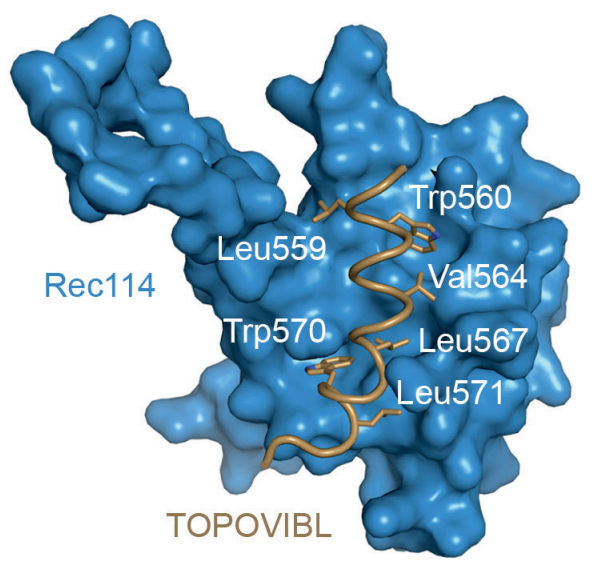

Rhinatrema bivittatum

Danio rerio

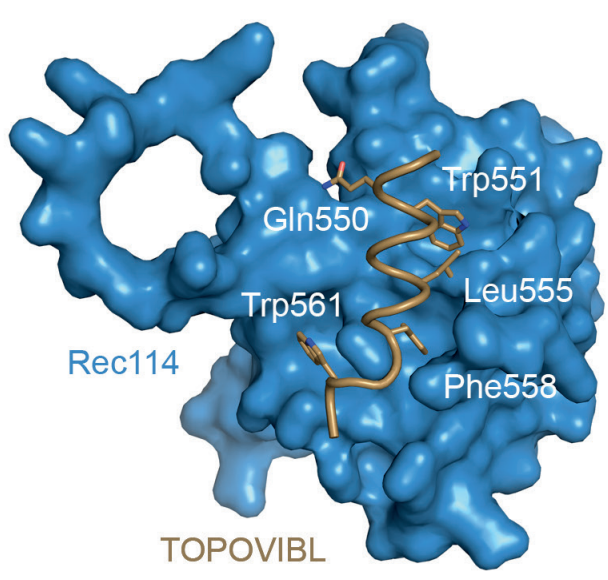

\section{Extended Data Fig.3}

Latimeria chalumnae
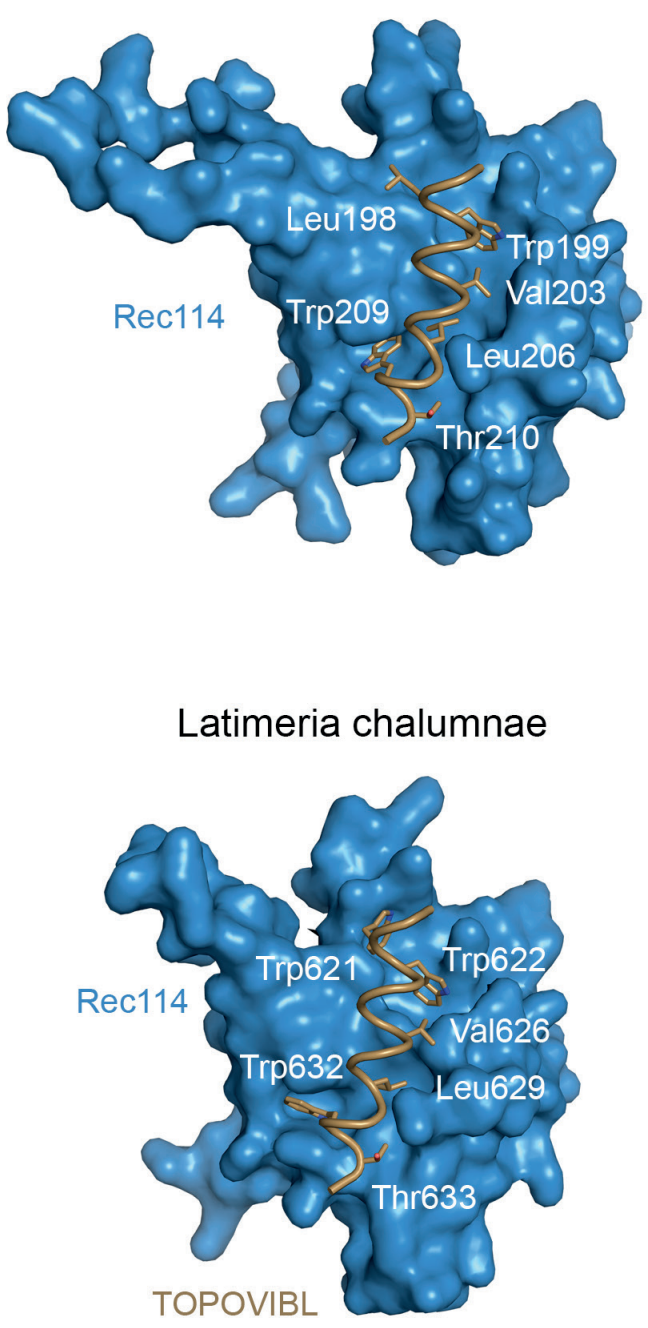

OPOVIBL 

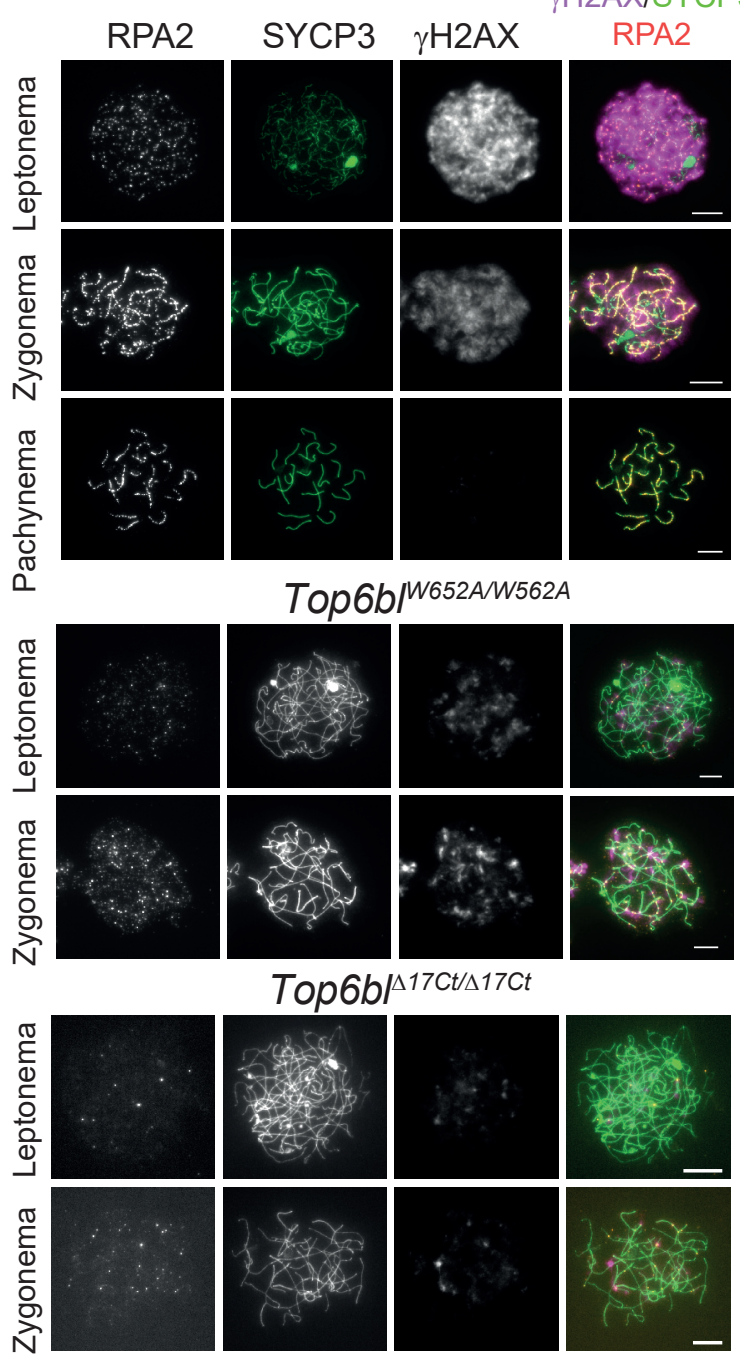

b

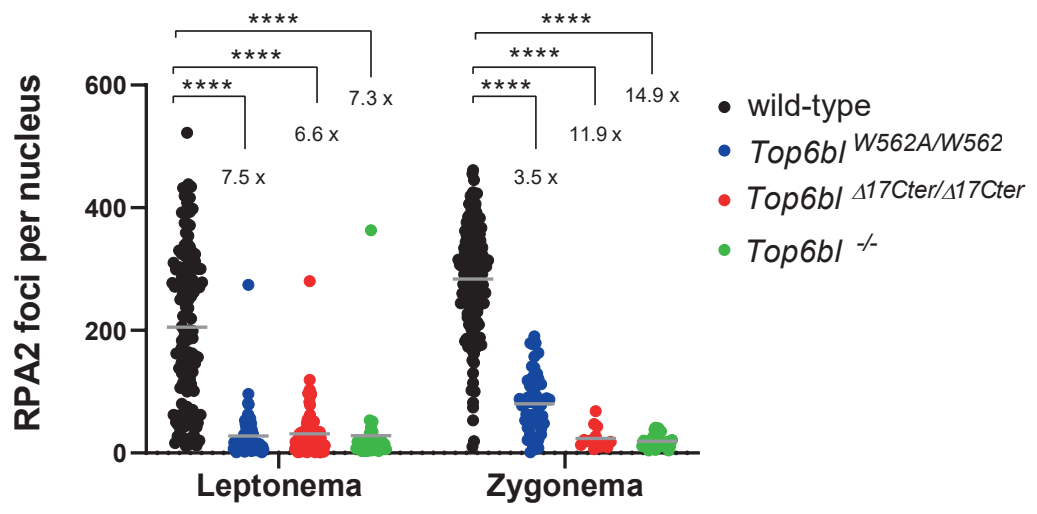

\section{Extended Data Fig.4}


bioRxiv preprint doi: https://doi.org/10.1101/2021.11.30.470517; this version posted December 2, 2021. The copyright holder for this

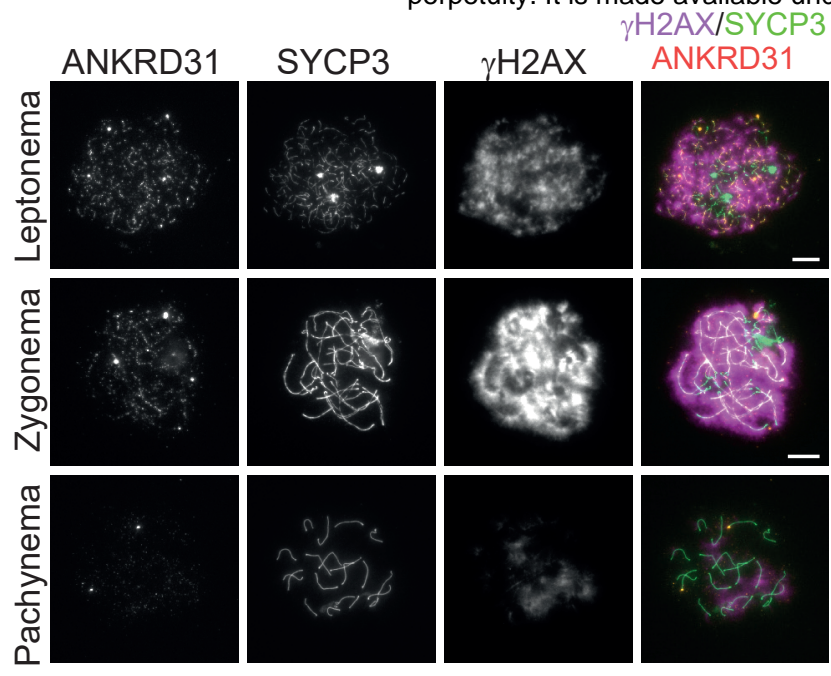

Top6b/W652A/W562A
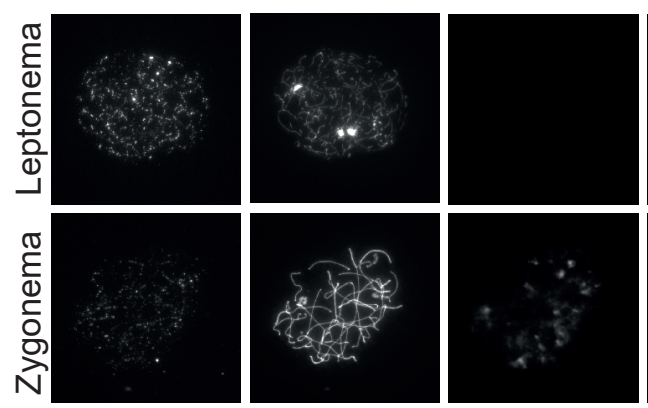

Top6b/17ct/A17Ct
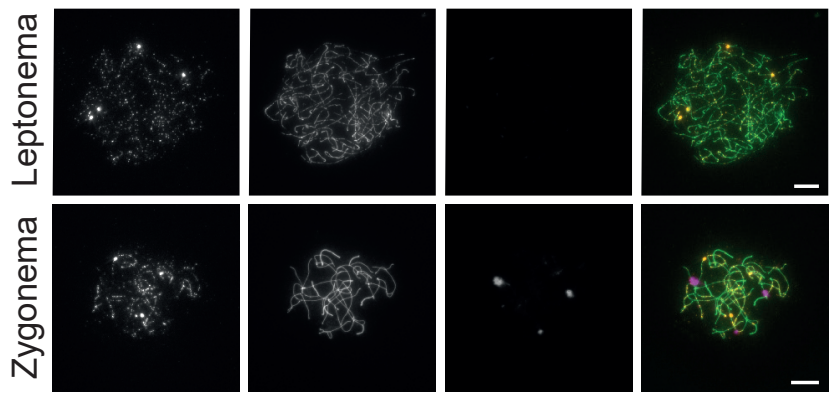

b

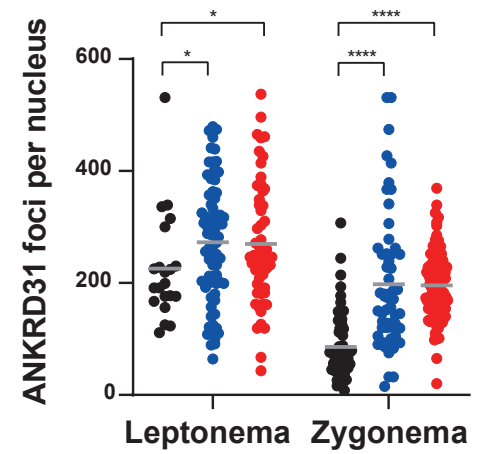

$\gamma \mathrm{H} 2 \mathrm{AX} / \mathrm{SYCP} 3$
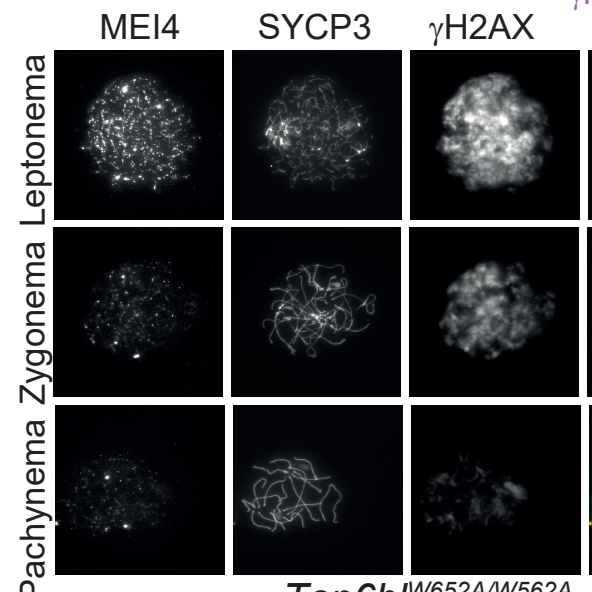
MEI4

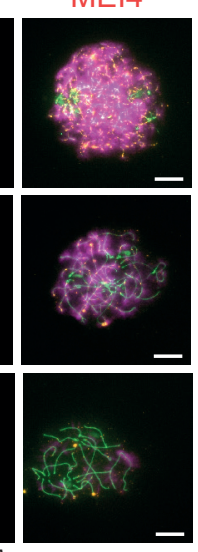

Top6b/W652A/W562A
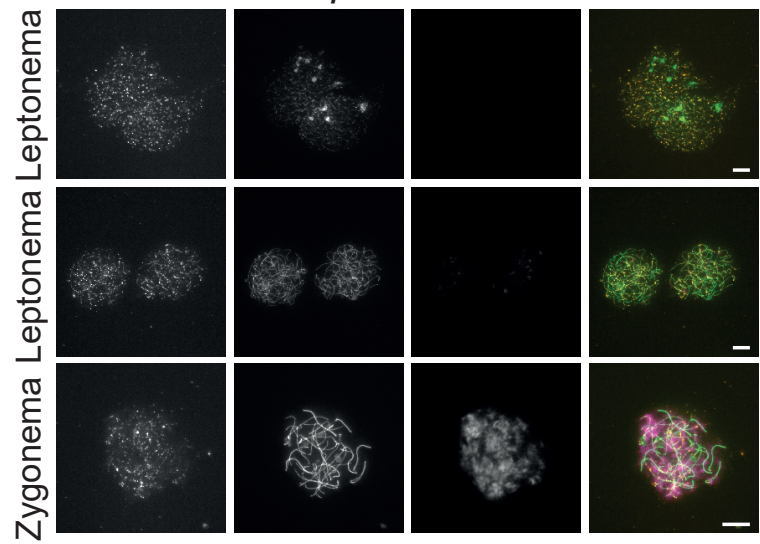

Top6b/ $/^{17 C t / \Delta 17 C t}$

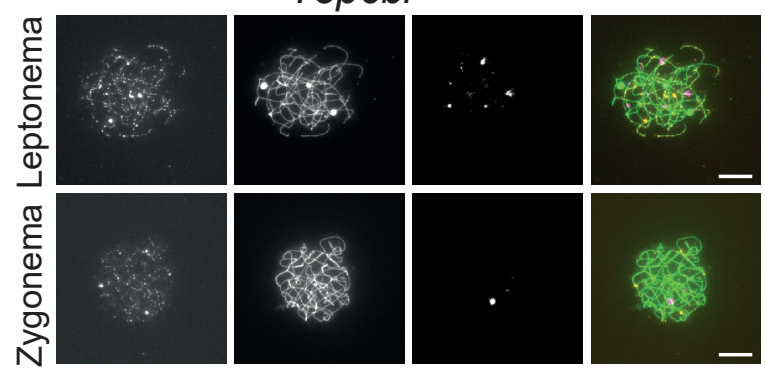

d

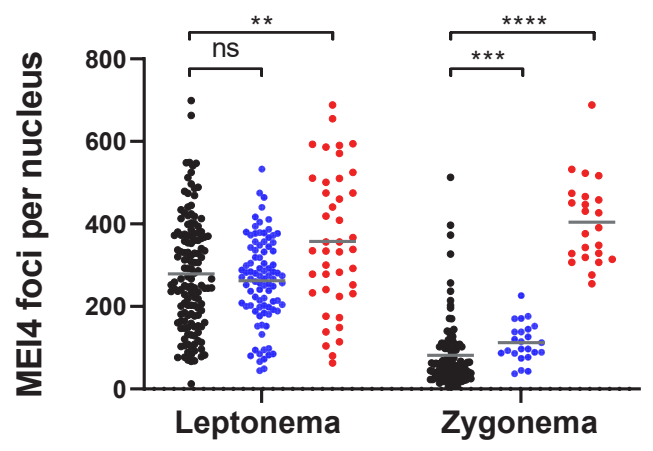

- wild type "Top6b/ ${ }^{\text {W562Aw562A }} \cdot$ Top6b/ ${ }^{\Delta 17 C t / \Delta 17 C t}$

\section{Extended Data Fig.5}




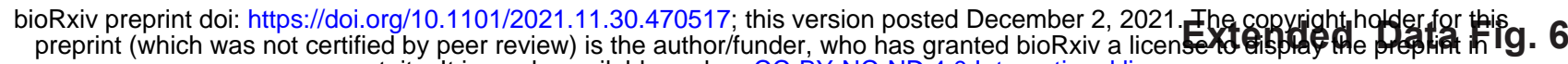
perpetuity. It is made available under aCC-BY-NC-ND 4.0 International license.

a wild-type

Top6b/W652A/W562A
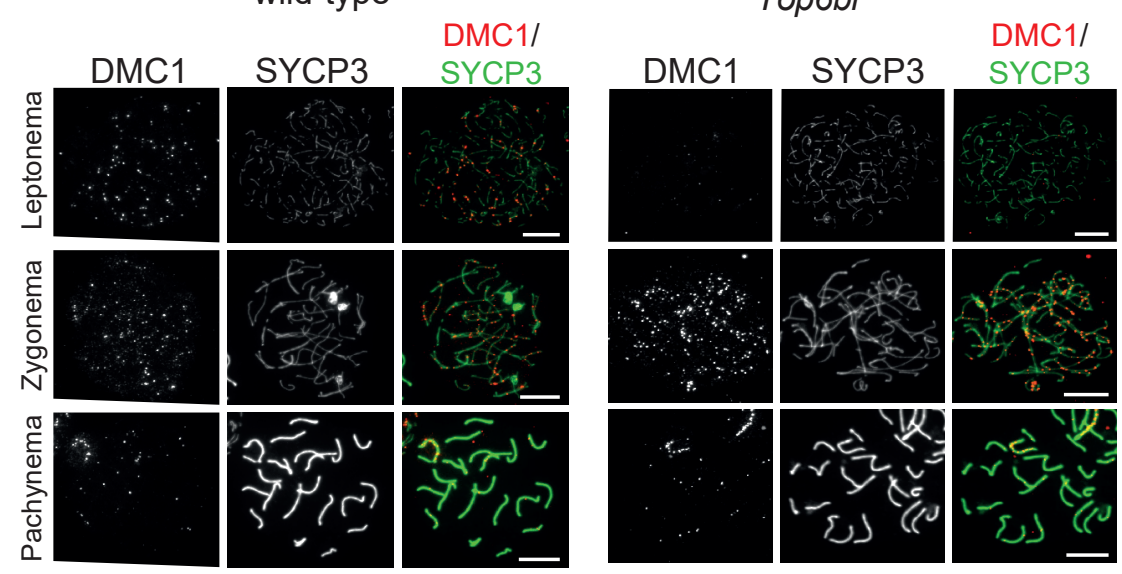

$T o p 6 b / 17 c t / 17 c t$
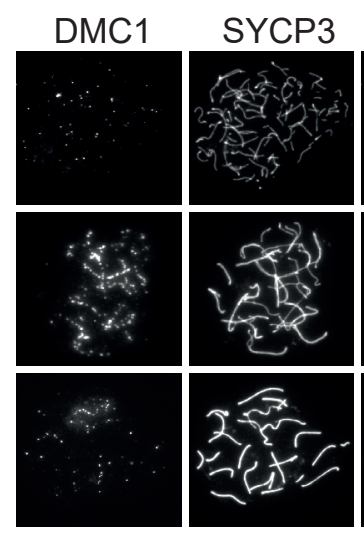

DMC1/

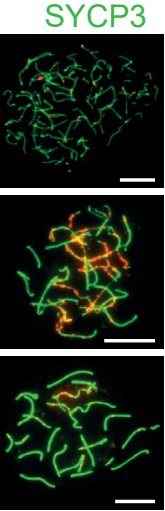

b

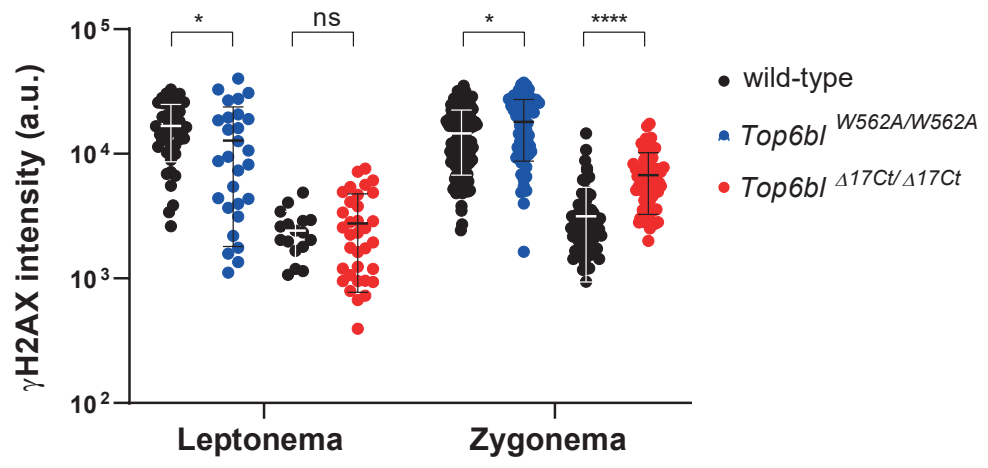

C

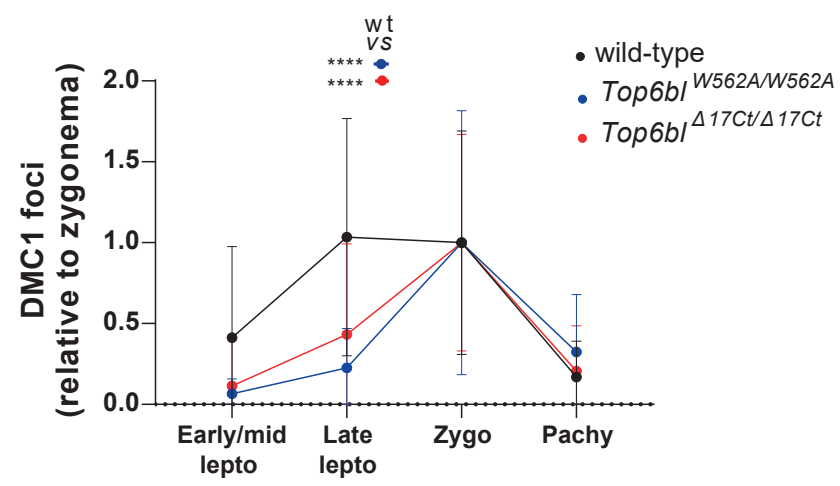

d

wild-type Top6b/W652Aw562A RPA2 SYCP3 $\gamma \mathrm{H} 2 \mathrm{AX} \underset{\mathrm{RPA} 2}{\gamma \mathrm{H} 2 \mathrm{AX} / \mathrm{SY}}$

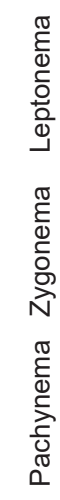
e

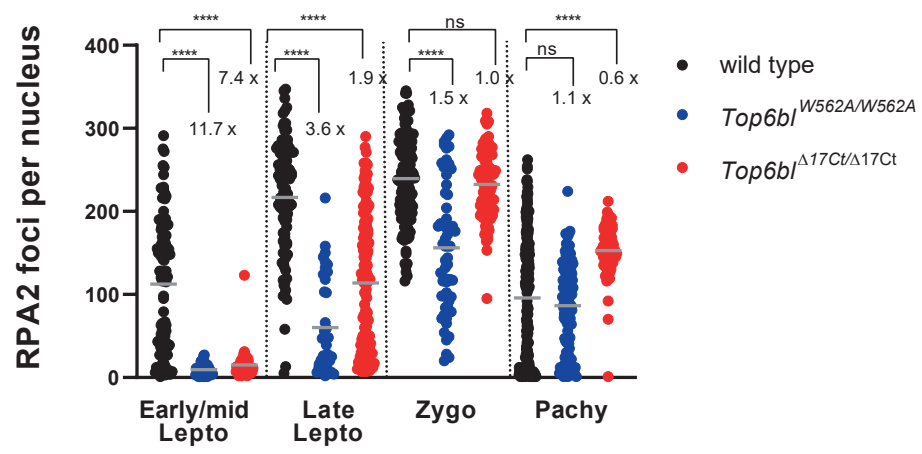

RPA2 SYCP3 $\gamma \mathrm{H} 2 \mathrm{AX}{ }^{\gamma \mathrm{H} 2 \mathrm{AX} / \mathrm{SYC} 3}$

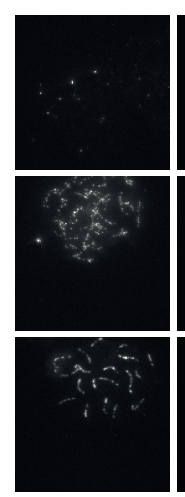

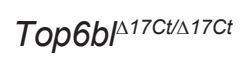

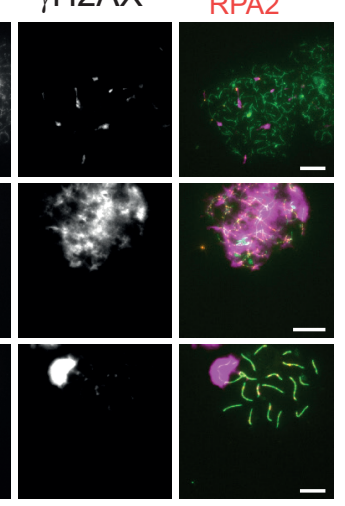

RPA2 SYCP3 $\gamma \mathrm{H} 2 \mathrm{AX}$

H2AXISYCP3
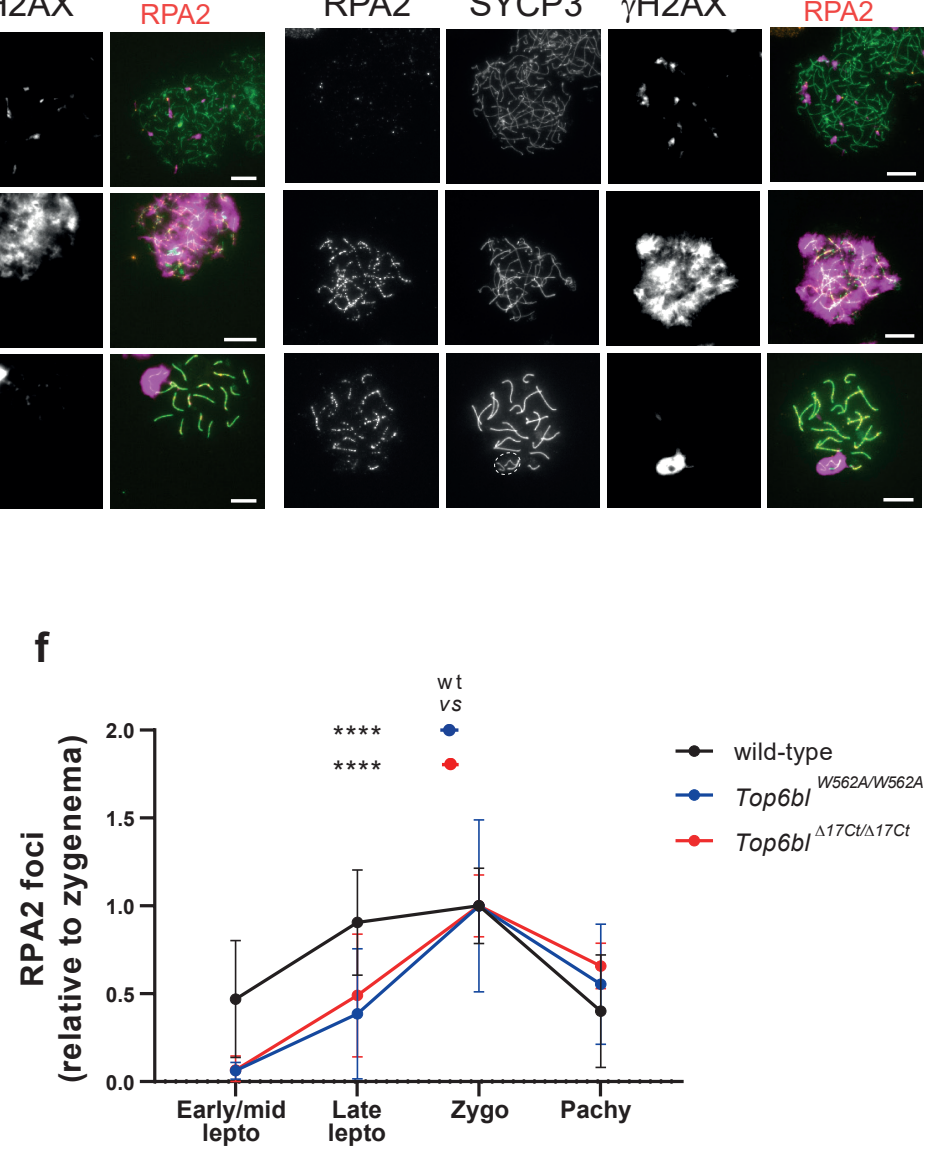


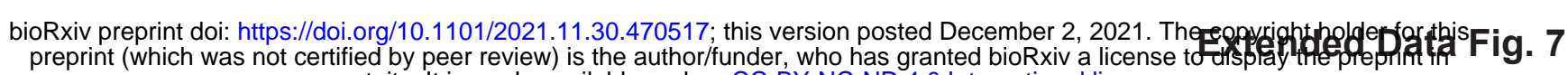
perpetuity. It is made available under aCC-BY-NC-ND 4.0 International license.

a

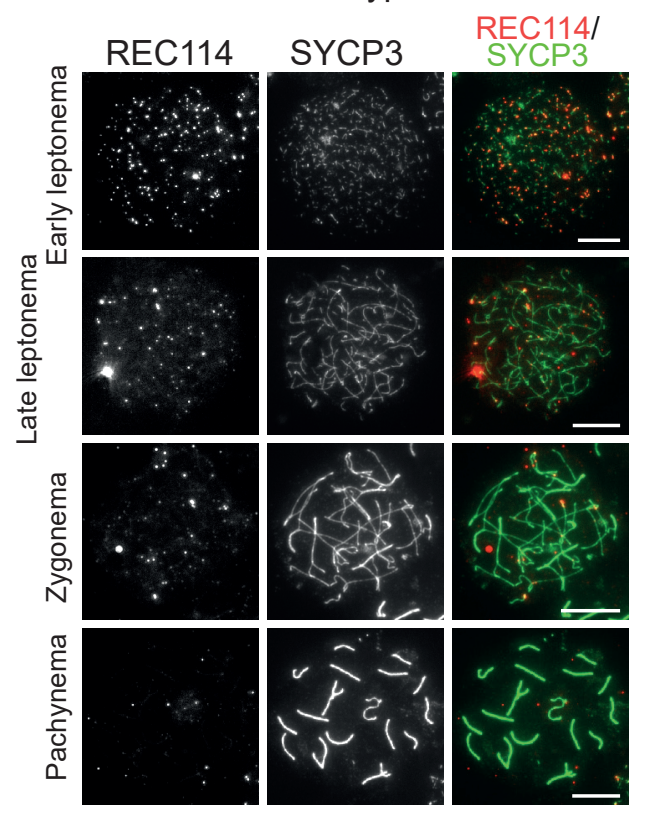

b

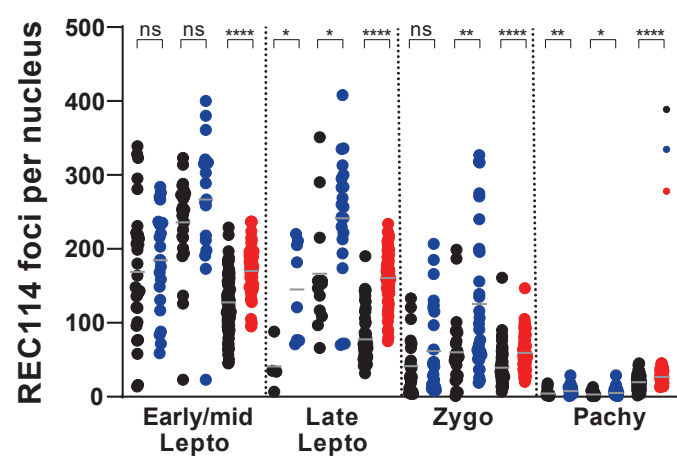

Top6b/W652A/W562A

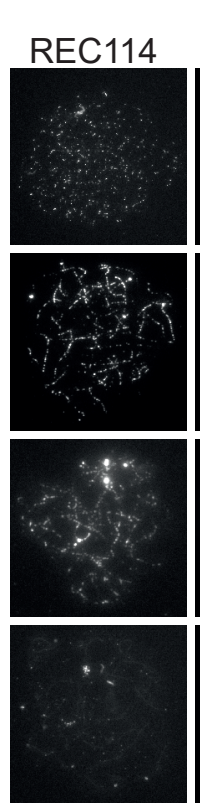

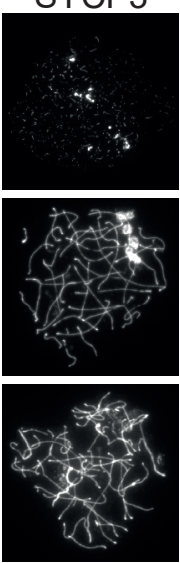

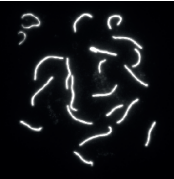

Top6b/ $17 \mathrm{17t} / \Delta 17 C t$

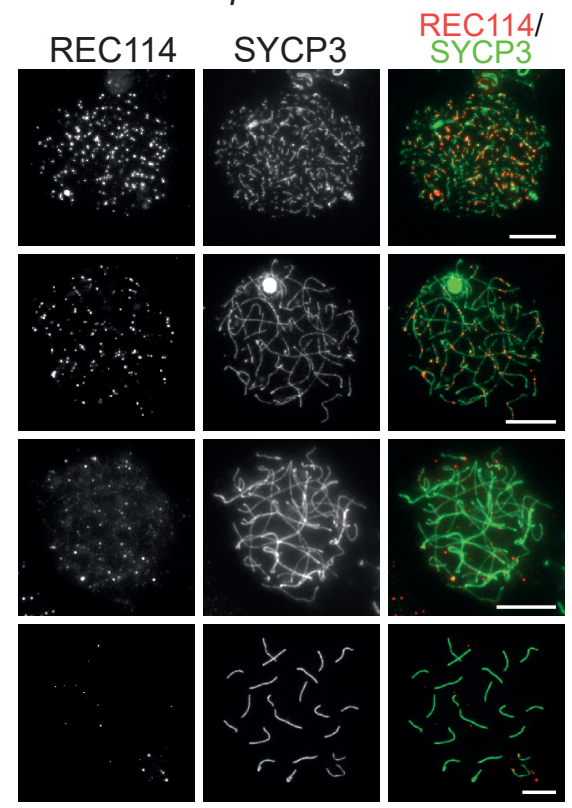

C

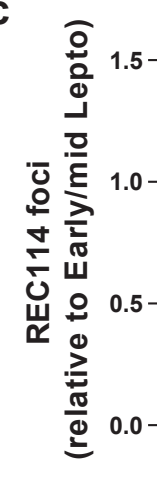

REC114/
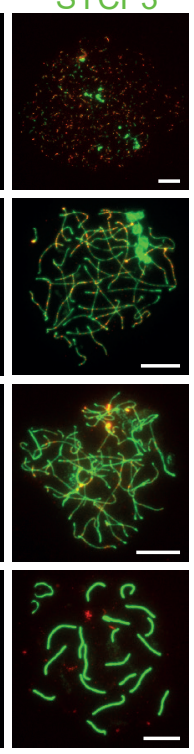

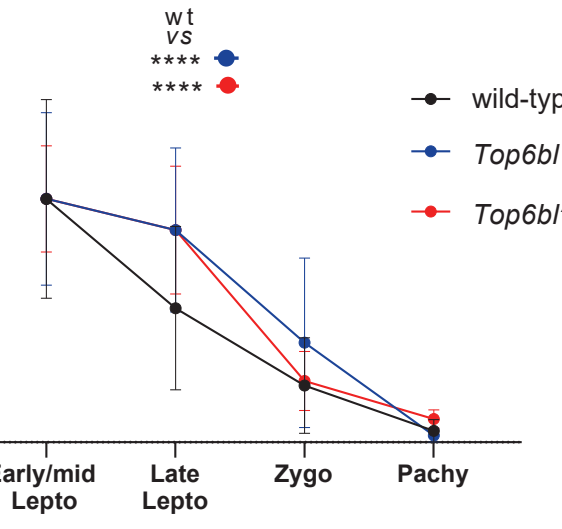

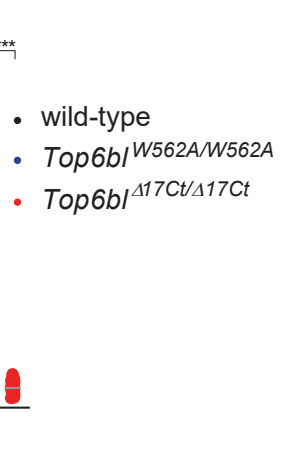

Top6b/W652A/W562A

Top $6 b /^{\Delta 17 c t / \Delta 17 c t}$

d wild-type

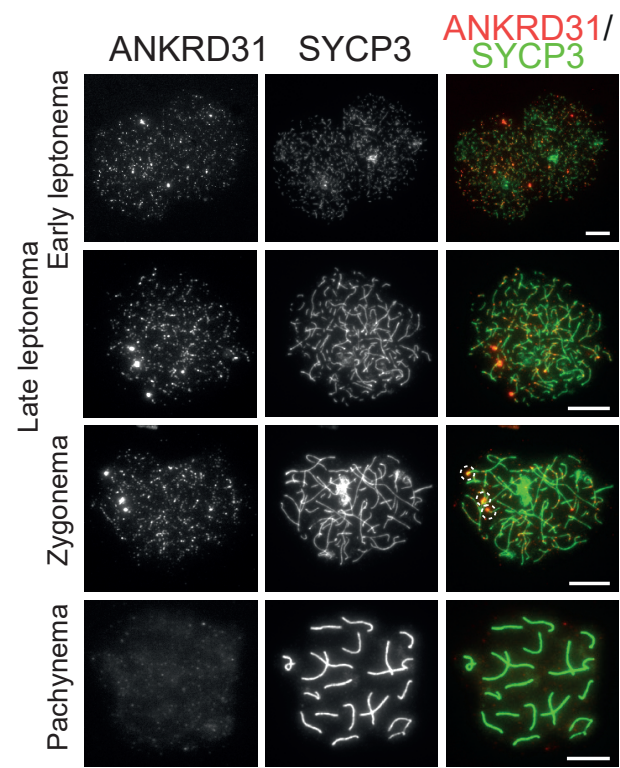

ANKRD31 SYCP3 ANKRD31/
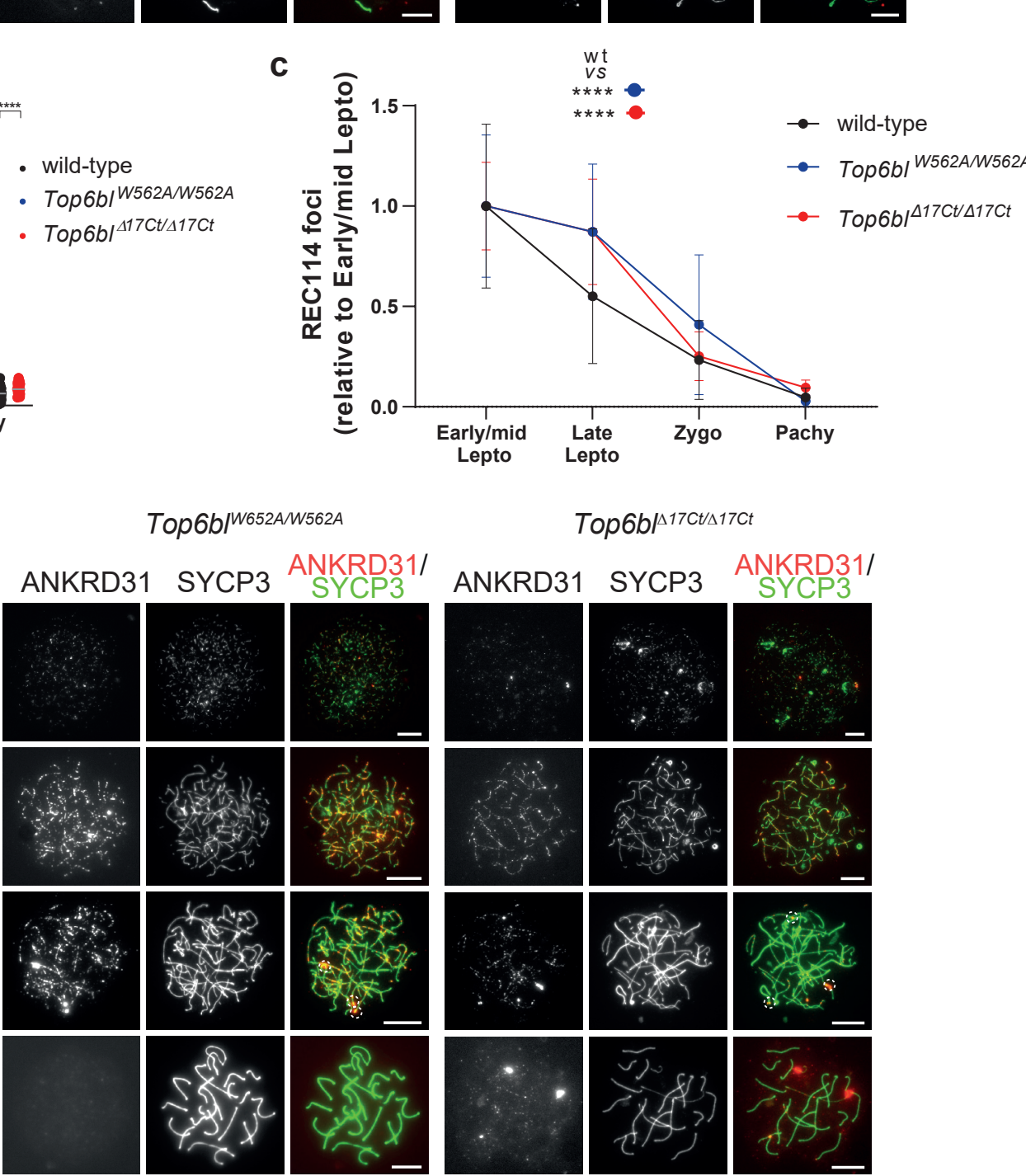
a

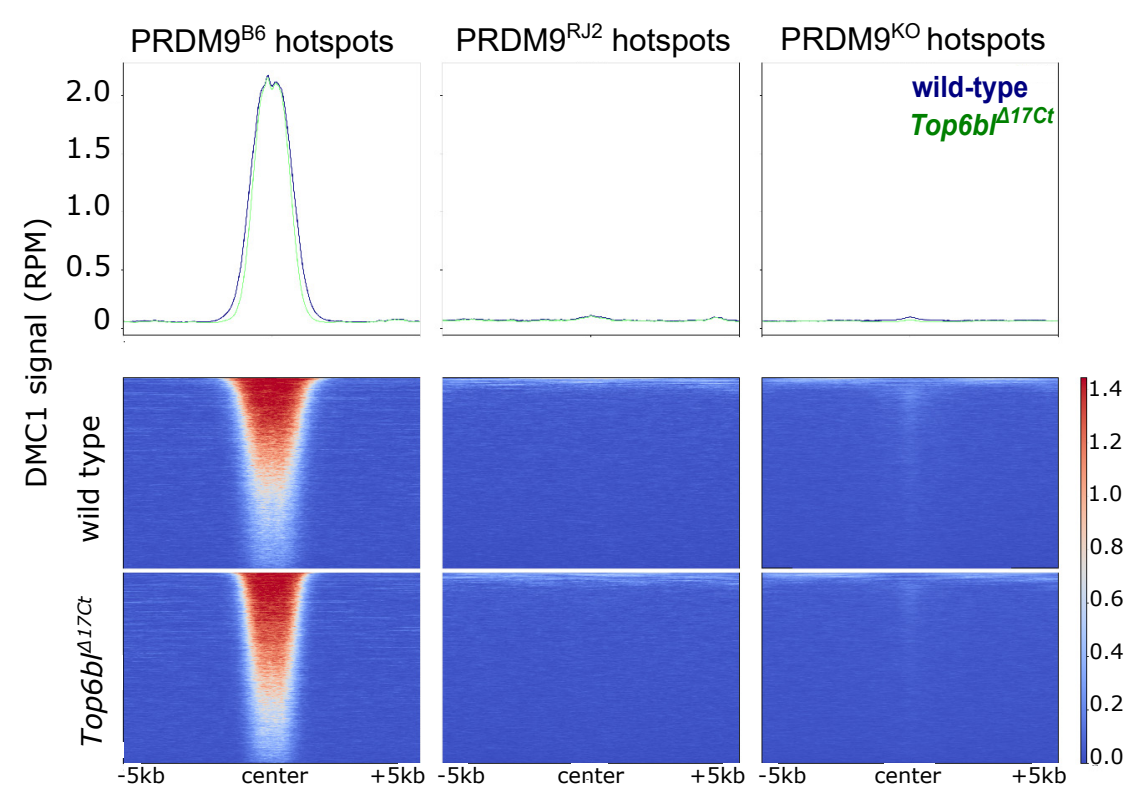

b

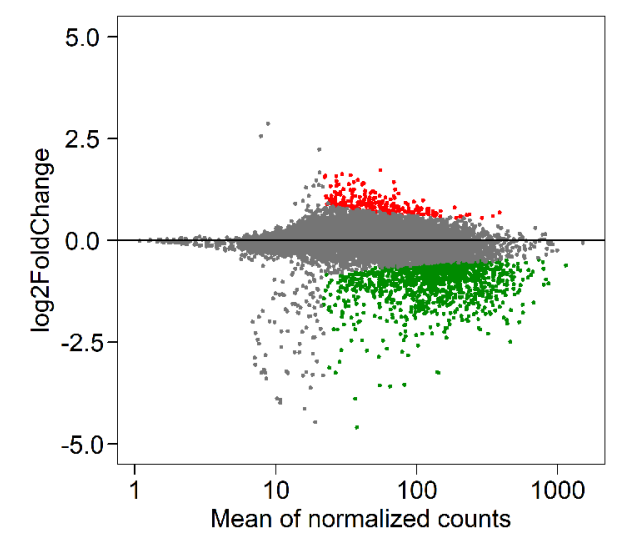

C

Unchanged $\square$ Decreased $\square$ Increased

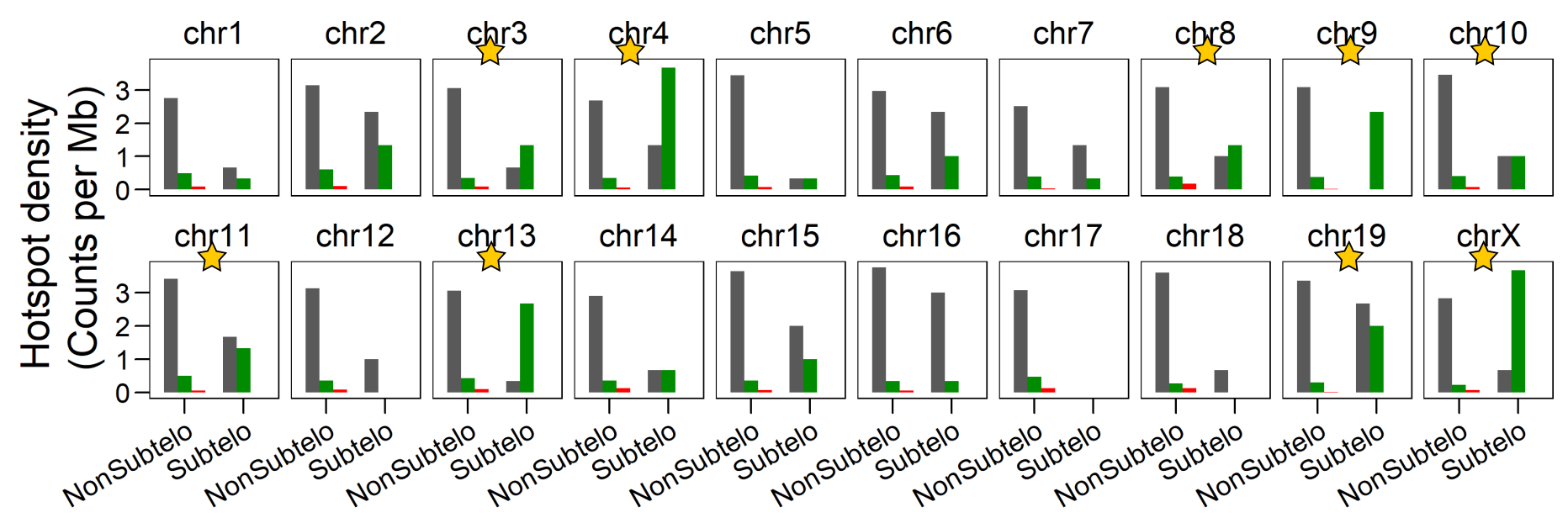

d

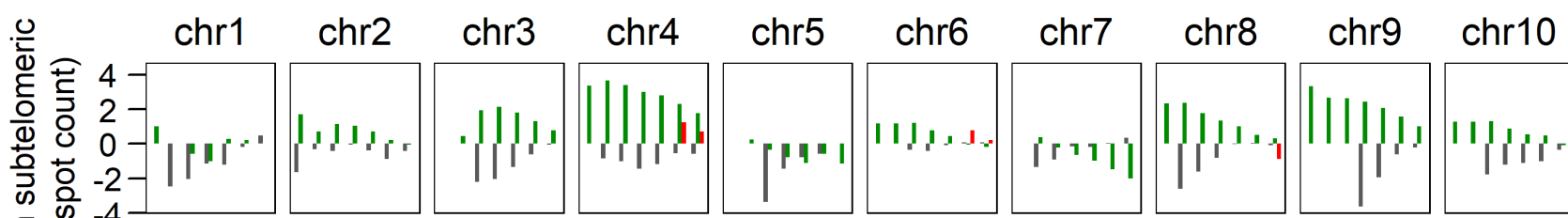

뭉 品

क

chr11 chr12

chr13 chr14
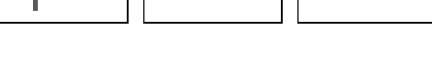

chr18 chr19 chrX

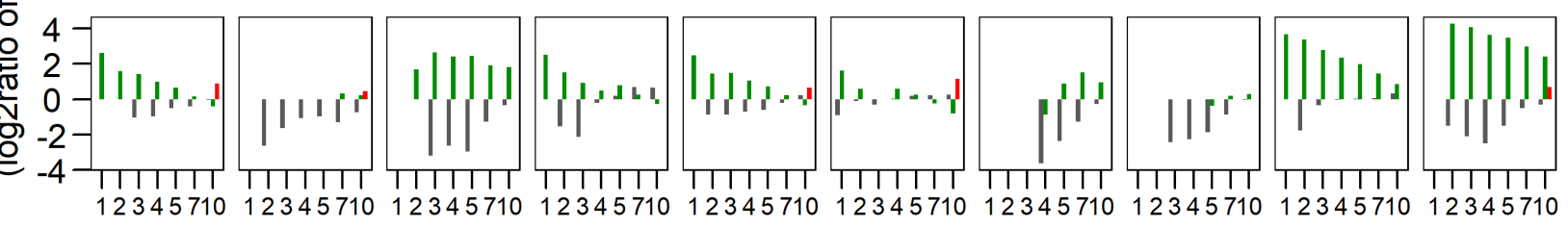

Subtelomeric size $(\mathrm{Mb})$ 
a

\begin{tabular}{|c|c|c|}
\hline Genotype & $\begin{array}{c}\text { Pachytene with } \\
\text { full autosomal } \\
\text { synapsis (\%) }\end{array}$ & Nuclei (n) \\
\hline$+/+$ & 76.6 & 77 \\
\hline$+/ T o p 6 b I^{W 562 A}$ & 86.7 & 30 \\
\hline wild-type & 83.2 & 107 \\
\hline Top $6 \mathrm{bl}^{\text {W562A }} /$ Top6bl ${ }^{\text {W62A }}$ & 86.4 & 81 \\
\hline Top6bl ${ }^{\text {W562A }} /$ Top 6 bl $^{\text {W562A }}$ & 96.3 & 27 \\
\hline Top6bl ${ }^{\text {W62A }}$ mutant & 88.9 & 108 \\
\hline$+/+$ & 91.0 & 79 \\
\hline +/Top6bl $\left.\right|^{\Delta 17 C t}$ & 72.2 & 54 \\
\hline wild-type & 88 & 133 \\
\hline Top6bl ${ }^{\Delta 17 C t} /$ Top6bl $I^{\Delta 17 C t}$ & 84.4 & 96 \\
\hline Top6bl ${ }^{\Delta 17 C t} /$ Top6bl ${ }^{\Delta 17 c t}$ & 85.2 & 108 \\
\hline Top6bl $^{\Delta 17 C t}$ mutant & 85 & 204 \\
\hline
\end{tabular}

b

wild-type

C
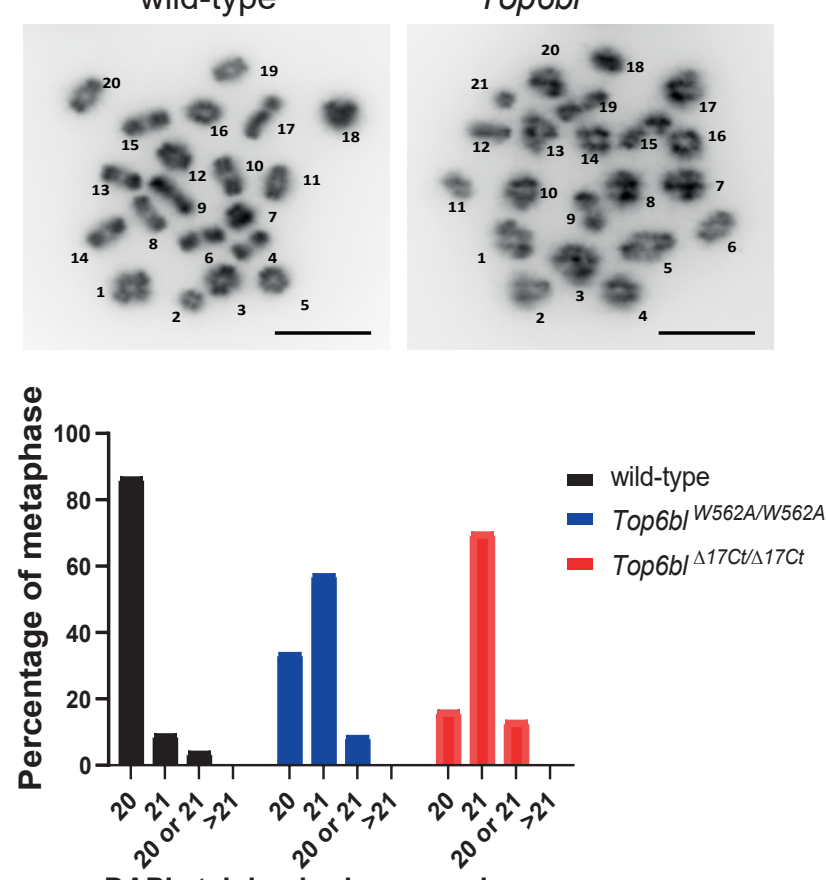

DAPI staining body per nucleus
Top6b/ ${ }^{\Delta 17 C t / \Delta 17 C t}$

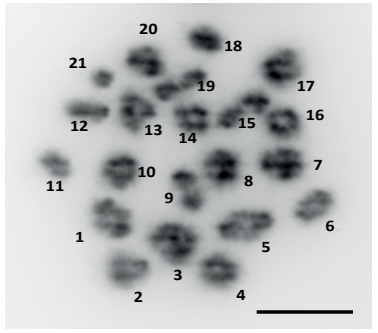

- Top6b/W562A/W562A

- Top6b/ ${ }^{\Delta 17 C t \Delta 17 C t}$ 
b

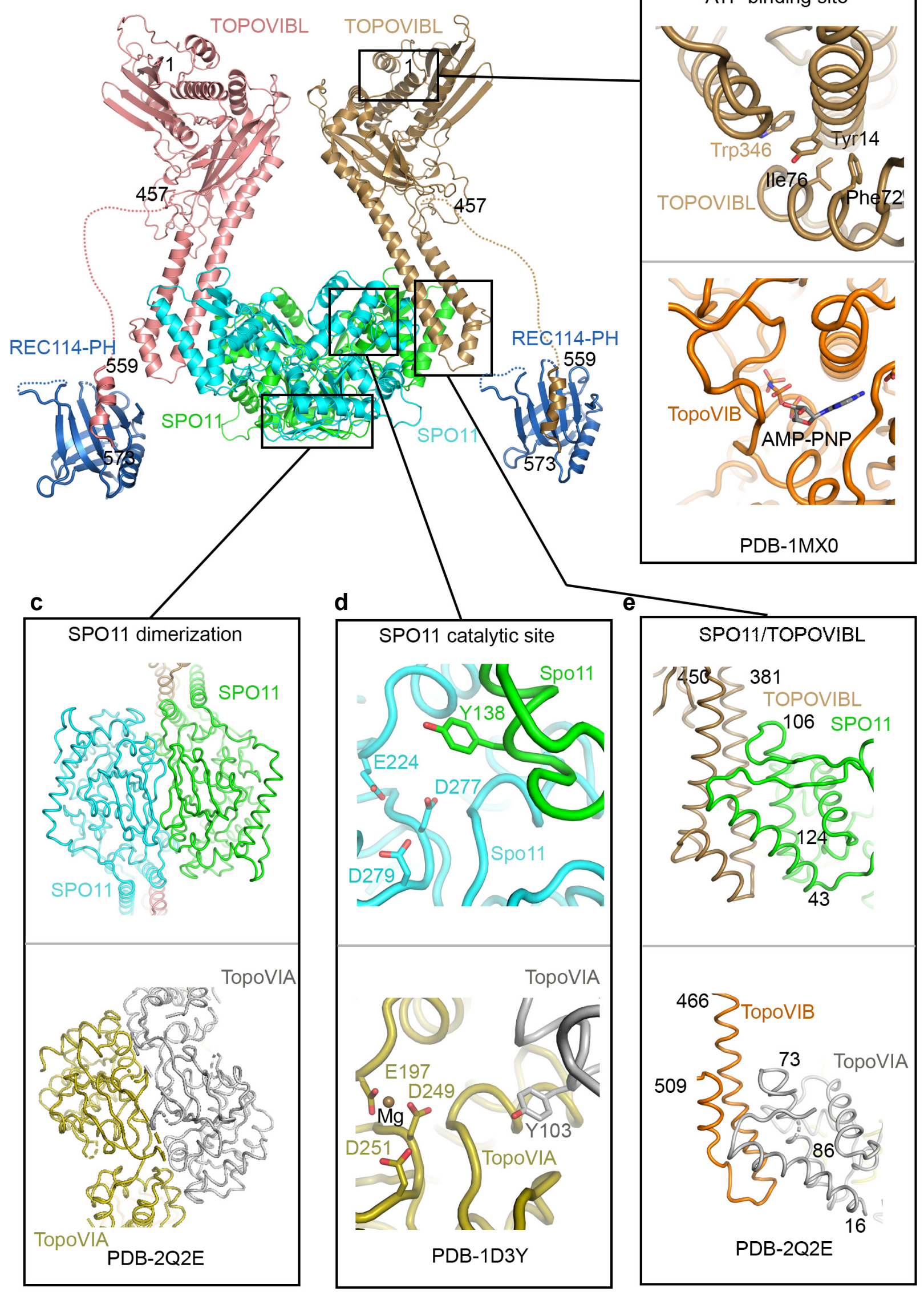

